\title{
Stationary and impulsive injection of electron beams in converging magnetic field
}

\author{
T. V. Siversky and V. V. Zharkova
}

\author{
Department of Mathematics, University of Bradford, Bradford BD7 1DP, UK \\ e-mail: taras.siversky@gmail.com
}

Received 16 April 2009 / Accepted 5 June 2009

\section{ABSTRACT}

\begin{abstract}
Aims. We study time-dependent precipitation of an electron beam injected into a flaring atmosphere with a converging magnetic field by considering collisional and Ohmic losses with anisotropic scattering and pitch angle diffusion. Two injection regimes are investigated: short impulse and stationary injection. The effects of converging magnetic fields with different spatial profiles are compared and the energy deposition produced by the precipitating electrons at different depths and regimes is calculated.

Methods. The time dependent Fokker-Planck equation for electron distribution in depth, energy and pitch angle was solved numerically by using the summary approximation method.

Results. Steady state injection was found to be established for beam electrons $0.07-0.2 \mathrm{~s}$ after the injection onset depending on the initial beam parameters. Energy deposition by a stationary beam is strongly dependent on a self-induced electric field but more weakly dependent on a magnetic field convergence. Energy depositions by short electron impulses are found to be insensitive to the self-induced electric field but are strongly affected by magnetic convergence. Short beam impulses are shown to produce sharp asymmetric hard X-ray bursts on a timescale of the order of tens of milliseconds, often observed in solar flares.
\end{abstract}

Key words. Sun: atmosphere - Sun: flares - Sun: X-rays, gamma rays - scattering - radiation mechanisms: non-thermal X-rays: bursts

\section{Introduction}

Observations of solar flares in hard X-rays provide vital information about scenarios in which accelerated electrons gain and deposit their energy into flaring atmospheres. The theory describing the generation of bremsstrahlung emission has progressed significantly in many directions by improving the mechanisms for emitting this radiation, e.g., considering relativistic bremsstrahlung cross-sections (Kontar et al. 2006), taking into account various aspects of the photospheric albedo effects, while deriving mean electron spectra from the observed bremsstrahlung photon spectra (Kontar et al. 2006). On the other hand, substantial improvements have also been made in solving the direct problem of electron precipitation into a flaring atmosphere by taking into account different mechanisms of electron energy losses: Coulomb collisions (Brown 1971; Brown et al. 2000) combined with deceleration by the self-induced electric field (Zharkova et al. 1995; Zharkova \& Gordovskyy 2006) in flaring atmospheres with strong temperature and density gradients derived from hydrodynamic solutions (Somov et al. 1981, 1982; Nagai \& Emslie 1984; Fisher et al. 1985).

Substantial progress in the quantitative interpretation of hard $\mathrm{X}$-ray emission has been achieved by using high temporal and spatial resolution observations carried out by the RHESSI payload (Lin et al. 2003). These observations infer the locations and shapes of hard X-ray sources on the solar disk, their temporal variations, and energy spectra evolution during the flare duration (Holman et al. 2003; Krucker et al. 2008). These observations are often accompanied by other observations (in microwaves (MW), EUV, and optical ranges), which identify a very close temporal correlation between HXR and MW, UV, and even optical emission (see for example, Kundu et al. 2004;
Fletcher et al. 2007; Grechnev et al. 2008. This has highlighted the further need for improvements in electron transport models, which can simultaneously account both temporarily and spatially for all these types of emission.

The RHESSI observations of double power law energy spectra, which exhibit flattening towards lower photon energies (Holman et al. 2003) leading to the soft-hard-soft temporal pattern of the photon spectra indices below $35 \mathrm{keV}$ (Grigis \& Benz 2006), highlighted the role of a self-induced electric field of beam electrons in their spectral flattening (Zharkova \& Gordovskyy 2006). The authors considered a stationary beam injection and naturally reproduced the spectral flattening by means of electron deceleration in the electric field, induced by beam electrons themselves. The flattening was shown to be proportional to the initial energy flux of beam electrons and their spectral indices (Zharkova \& Gordovskyy 2006). The soft-hard-soft pattern in photon spectra above can then be easily reproduced by a triangular increase and decrease in the beam energy flux within a time interval of a few seconds, which was often observed both by RHESSI (Lin et al. 2003), and by the previous SMM mission (Kane et al. 1980). Furthermore, numerous observations of solar flares by SMM, TRACE, and RHESSI suggest that the areas of flaring loops decrease and, thus, their magnetic fields increase with the depth of the solar atmosphere (Lang et al. 1993; Brosius \& White 2006; Kontar et al. 2008). This increase in magnetic field can act as a magnetic mirror for the precipitating electrons that, in addition to the effect of self-induced electric field, forces them to return to their source in the corona.

In the present paper, we propose two models of magnetic field variations with depth. One model fits measurements of the magnetic field in the corona (Brosius \& White 2006) and 
chromosphere (Kontar et al. 2008), another shows the exponential increase in the magnetic field from the corona to the upper chromosphere, while retaining a constant value in the lower chromosphere. The outcome is compared with those of the two other models proposed earlier: the first one (Leach \& Petrosian 1981), where the authors assumed that the magnetic column depth scale $\partial \ln B / \partial s$ is constant implying that there is an exponential magnetic field increase with column depth, and the second one (McClements 1992) including a parabolic increase in the magnetic field with linear depth.

The temporal intervals of impulsive increases in HXR emission also vary from very short tens of milliseconds (Kiplinger et al. 1983; Charikov et al. 2004) to tens of minutes as often observed by RHESSI (Holman et al. 2003). This encouraged us to revise the electron transport models and consider solutions of a time-dependent Fokker-Planck equation for different timescales of beam injection (milliseconds, seconds, and minutes). The electron transport, in turn, can also be deaccelerated by anisotropic scattering of beam electrons in this self-induced electric field enhanced by the magnetic mirroring of the same electrons in converging magnetic loops. The additional delay can be caused by the particle diffusion in pitch angles and energy, which can significantly extend the electron transport time into deeper atmospheric layers where they are fully thermalised.

We also apply the time dependent Fokker-Planck equation to compare the solutions for electron precipitation for stationary and impulsive injection and their effect on resulting hard X-ray emission and ambient plasma heating for different parameters of beam electrons. We also investigate these Fokker-Planck solutions for the different models of a converging magnetic field by taking into account all the mechanisms of energy loss (collisions and Ohmic losses) and anisotropic scattering but without diffusion of energy.

The problem is formulated in Sect. 2, and the method of solution is described in Sect. 3. The stationary injection into a flaring atmosphere with different magnetic field convergence and collisional plus Ohmic losses with anisotropic scattering is considered in Sect. 4, and the impulsive injection on short timescales below tens milliseconds is considered in Sect. 5. The discussion and conclusions are drawn in Sect. 6.

\section{Problem formulation}

\subsection{The Fokker-Planck equation}

We consider a one-dimensional beam of high energy electrons injected into the solar atmosphere. The beam electron velocity distribution $f$, as a function of time $t$, depth $l$, velocity $V$, and pitch angle between the velocity and the magnetic field $\theta$, can be found by solving the Fokker-Planck equation (Diakonov \& Somov 1988; Zharkova et al. 1995)

$$
\begin{aligned}
\frac{\partial f}{\partial t}+ & V \cos \theta \frac{\partial f}{\partial l}-\frac{e \mathcal{E}}{m_{\mathrm{e}}} \cos \theta \frac{\partial f}{\partial V}-\frac{e \mathcal{E}}{m_{\mathrm{e}} V} \sin ^{2} \theta \frac{\partial f}{\partial \cos \theta}= \\
& \frac{1}{V^{2}} \frac{\partial}{\partial V}\left(v V^{3} f\right)+v \frac{\partial}{\partial \cos \theta}\left(\sin ^{2} \theta \frac{\partial f}{\partial \cos \theta}\right) \\
+ & \frac{V \sin ^{2} \theta}{2} \frac{\partial \ln B}{\partial l} \frac{\partial f}{\partial \cos \theta},
\end{aligned}
$$

where the collisional rate $v$ is given by

$$
v=n_{\mathrm{p}}(l) \frac{2 \pi e^{4} \ln \Lambda}{m_{\mathrm{e}}^{2} V^{3}},
$$

$\mathcal{E}$ is the self-induced electric field, $B$ is the background magnetic field, $n_{\mathrm{p}}(l)$ is the density of the ambient plasma, $\ln \Lambda$ is the Coulomb logarithm, and $e$ and $m_{\mathrm{e}}$ are the electron charge and mass, respectively. In our study, we assume that the Coulomb logarithm is constant, i.e., $\ln \Lambda \approx 20$.

We introduce the following dimensionless variables:

$\tau=t \frac{2 \pi e^{4} n_{0} \ln \Lambda}{\sqrt{2 m_{\mathrm{e}}} E_{0}^{3 / 2}}$

$s=\xi \frac{\pi e^{4} \ln \Lambda}{E_{0}^{2}}$,

$z=\frac{E}{E_{0}}=\frac{m_{\mathrm{e}} V^{2}}{2 E_{0}}$

$\mu=\cos \theta$,

$\varepsilon=\mathcal{E} \frac{E_{0}}{2 \pi e^{3} n_{0} \ln \Lambda}$,

$n=\frac{n_{\mathrm{p}}}{n_{0}}$

where $\xi$ is the column depth

$\xi=\int_{0}^{l} n_{\mathrm{p}}\left(l^{\prime}\right) \mathrm{d} l^{\prime}$

$E_{0}=12 \mathrm{keV}$ is the lower cut-off energy and $n_{0}=10^{10} \mathrm{~cm}^{-3}$. In dimensionless variables, Eq. (1) takes the form

$$
\begin{gathered}
\frac{\partial f}{\partial \tau}+n \sqrt{z} \mu \frac{\partial f}{\partial s}-2 \varepsilon \mu \sqrt{z} \frac{\partial f}{\partial z}-\varepsilon \frac{1-\mu^{2}}{\sqrt{z}} \frac{\partial f}{\partial \mu}= \\
n \frac{1}{\sqrt{z}} \frac{\partial f}{\partial z}+n \frac{1-\mu^{2}}{2 z^{3 / 2}} \frac{\partial^{2} f}{\partial \mu^{2}}-n \frac{\mu}{z^{3 / 2}} \frac{\partial f}{\partial \mu} \\
+n \frac{\left(1-\mu^{2}\right) \sqrt{z}}{2} \alpha_{B} \frac{\partial f}{\partial \mu}
\end{gathered}
$$

where $\alpha_{B}$ is the magnetic convergence parameter defined to be

$\alpha_{B}=\frac{\partial \ln B}{\partial s}$.

As shown by van den Oord (1990), after a few collisional times from the start of the beam injection, the background plasma fully compensates for the charge and current deposited by the beam. In our model, we assume that the beam current is always compensated, i.e., $j_{\mathrm{b}}+j_{\mathrm{p}}=0$, where $j_{\mathrm{b}}$ and $j_{\mathrm{p}}$ are the beam and plasma currents, respectively. We note that a beam current $j_{\mathrm{b}}$ includes electrons with $\mu<0$, i.e., those that move back to source, which can significantly decrease the compensation current $j_{\mathrm{p}}$. By applying Ohm's law for $j_{\mathrm{p}}$ and taking into account that $j_{\mathrm{p}}=-j_{\mathrm{b}}$, we can then find the self-induced electric field (McClements 1992)

$\varepsilon(\tau, s)=\frac{1}{\sigma(s)} \int_{z_{\min }}^{z_{\max }} \mathrm{d} z \int_{-1}^{1} \mathrm{~d} \mu z \mu f(\tau, s, z, \mu)$,

where the dimensionless conductivity is

$\sigma(s)=1.97 \sqrt{2 \pi} \frac{3}{4} \frac{n_{0}(k T(s))^{3 / 2}}{\sqrt{m_{\mathrm{e}}} F_{0}}$,

and $F_{0}=10^{10} \mathrm{erg} \mathrm{cm}^{-2} \mathrm{~s}^{-1}$ is the normalisation factor for the energy flux of the beam. The ambient plasma is assumed to be 


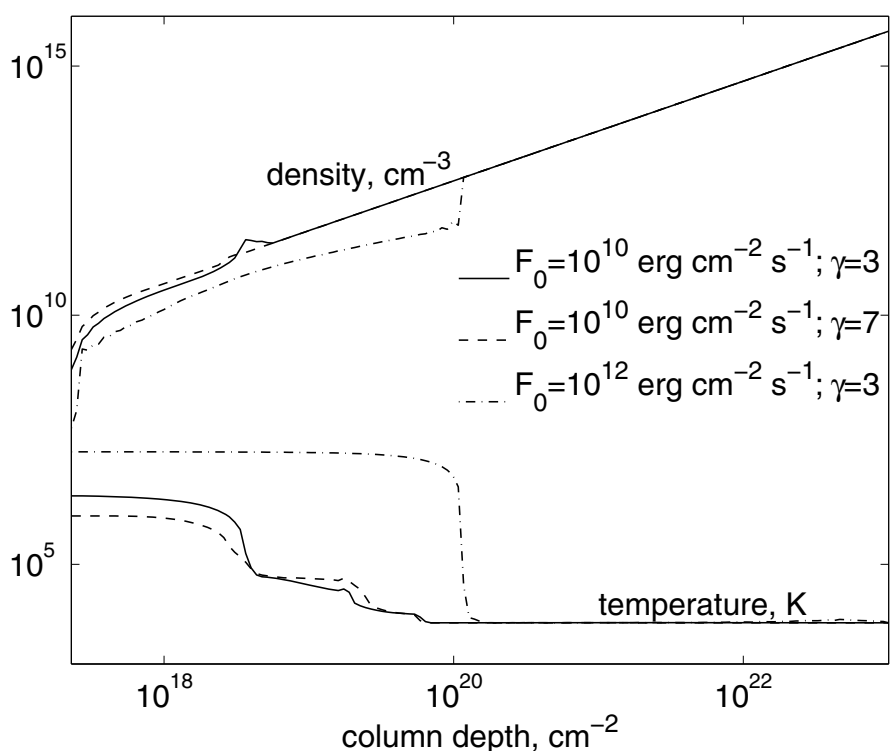

Fig. 1. Density and temperature of the ambient plasma calculated by the hydro-dynamic model (Zharkova \& Zharkov 2007) for different beam energy flux, $F_{0}$, and power law index, $\gamma$, of the beam electron distribution (see Eq. (15)).

preheated and its density, $n$, and temperature, $T$, as functions of the column depth, $s$, are calculated using the hydrodynamic model (Zharkova \& Zharkov 2007). The profiles $n(s)$ and $T(s)$ for different beam parameters are shown in Fig. 1. These profiles do not change in time, since the thermal conduction processes occur on much longer timescales than the precipitation processes studied here.

The electron beam and the plasma return current can be a subject of various plasma instabilities. For example, Zharkova \& Kobylinskij (1992) demonstrated that a beam with energy flux higher than $10^{11} \mathrm{erg} \mathrm{cm}^{-2} \mathrm{~s}^{-1}$ can be unstable with respect to the ion-sound waves. However, these instabilities are beyond the scope of the current paper and will be considered in forthcoming studies.

\subsection{Initial and boundary conditions}

There are no beam electrons before the injection starts, thus, the initial condition is

$f(\tau=0, s, z, \mu)=0$.

The boundary condition at $s=s_{\min }=2.08 \times 10^{-3}$ (or $2.29 \times 10^{17} \mathrm{~cm}^{-2}$ ) corresponds to the injected beam distribution

$f\left(\tau, s=s_{\min }, z, \mu>0\right)=f_{\mathrm{n}} \psi(\tau) \frac{z^{\delta-1}}{z^{\delta+\gamma}+1} \exp \left(-\frac{(1-\mu)^{2}}{\Delta \mu^{2}}\right)$

where $\Delta \mu$ is the initial pitch angle dispersion and $\psi(\tau)$ determines the time variation in the beam. If the energy is much higher than the lower cut-off energy, $z \gg 1$, the distribution is a power law with index $-\gamma-1$, and thus, the flux spectrum $(\sim z f)$ is given by a power law with index $\gamma$. In the opposite case, $z \ll 1$, the distribution is a power law with index $\delta-1$. The low energy index $\delta$ is chosen to be 10 (see, e.g., Zharkova \& Gordovskyy 2005a), while for the high energy index, two values, 3 and 7, are considered. We select $f_{\mathrm{n}}$, the normalisation coefficient, so that the energy flux of the injecting electron beam

$$
F\left(s=s_{\min }\right)=F_{0} \int_{z_{\min }}^{z_{\max }} \mathrm{d} z \int_{-1}^{1} \mathrm{~d} \mu z^{2} \mu f\left(s=s_{\min }, z, \mu\right)
$$

is equal to some preset value $F_{\text {top }}$, where $F_{0}=10^{10} \mathrm{erg} \mathrm{cm}^{-2} \mathrm{~s}^{-1}$ is the normalisation factor of the energy flux. At large depth, $s=s_{\max }=9.17 \times 10^{2}$ (or $1.01 \times 10^{23} \mathrm{~cm}^{-2}$ ), the number of electrons in the beam is assumed to be negligibly small, thus the corresponding boundary condition is

$f\left(\tau, s=s_{\max }, z, \mu<0\right)=0$.

The distribution function is calculated for the following range of energies: $z_{\min } \leq z \leq z_{\max }$, where $z_{\min }=0.1$ (or $1.2 \mathrm{keV}$ ) and $z_{\max }=100$ (or $1.2 \mathrm{MeV}$ ). The boundary conditions on energy are

$$
\begin{aligned}
& \frac{\partial f\left(\tau, s, z=z_{\min }, \mu\right)}{\partial z}=0, \\
& \frac{\partial f\left(\tau, s, z=z_{\max }, \mu\right)}{\partial z}=0 .
\end{aligned}
$$

The boundary conditions on pitch angle are (McClements 1990)

$$
\begin{gathered}
\frac{\partial f(\tau, s, z, \mu=1)}{\partial \mu}=0, \\
\frac{\partial f(\tau, s, z, \mu=-1)}{\partial \mu}=0 .
\end{gathered}
$$

\subsection{Integral characteristics of the electron distribution in the beam}

Before numerically solving Eq. (10), we define the following quantities of the electron beam that we propose to calculate: beam density (in $\mathrm{cm}^{-3}$ ),

$n_{\mathrm{b}}(\tau, s)=F_{0} \sqrt{\frac{m_{\mathrm{e}}}{2 E_{0}^{3}}} \int_{z_{\min }}^{z_{\max }} \mathrm{d} z \int_{-1}^{1} \mathrm{~d} \mu \sqrt{z} A(s) f(\tau, s, z, \mu) ;$

differential particle flux spectrum (in $\mathrm{erg}^{-1} \mathrm{~cm}^{-2} \mathrm{~s}^{-1}$ ),

$\mathcal{F}_{\mathrm{n}}(\tau, s, z)=\frac{F_{0}}{2 E_{0}^{2}} \int_{-1}^{1} \mathrm{~d} \mu z A(s) f(\tau, s, z, \mu) ;$

mean particle flux spectrum (in $\mathrm{erg}^{-1} \mathrm{~cm}^{-2} \mathrm{~s}^{-1}$ ) (Brown et al. 2003),

$\left\langle\mathcal{F}_{\mathrm{n}}\right\rangle(\tau, z)=\frac{F_{0}}{E_{0}^{2}} \frac{\int_{s_{\min }}^{s_{\max }} \mathrm{d} s \int_{-1}^{1} \mathrm{~d} \mu n^{-1}(s) A(s) z f(\tau, s, z, \mu)}{2 \int_{s_{\min }}^{s_{\max }} n^{-1}(s) \mathrm{d} s} ;$

angle distribution (in arbitrary units),

$\frac{\mathrm{d} N_{\mathrm{b}}(\tau, \mu)}{\mathrm{d} \mu}=\int_{s_{\min }}^{s_{\max }} \mathrm{d} s \int_{z_{\min }}^{z_{\max }} \mathrm{d} z n^{-1}(s) A(s) \sqrt{z} f(\tau, s, z, \mu)$ 
and energy deposition (or heating function) of the beam (in $\operatorname{erg} \mathrm{cm}^{-3} \mathrm{~s}^{-1}$ ),

$I(\tau, s)=\frac{F_{0} n_{0}}{E_{0}} n(s) A(s) \int_{z_{\min }}^{z_{\max }} \mathrm{d} z \int_{-1}^{1} \mathrm{~d} \mu\left(-\frac{\mathrm{d} z}{\mathrm{~d} s}\right) \mu z f(\tau, s, z, \mu)$,

where $\mathrm{d} z / \mathrm{d} s$ is the electron's energy losses with depth, which can be estimated as (Emslie 1980)

$\frac{\mathrm{d} z}{\mathrm{~d} s}=\left(\frac{\mathrm{d} z}{\mathrm{~d} s}\right)_{\mathrm{c}}+\left(\frac{\mathrm{d} z}{\mathrm{~d} s}\right)_{\mathrm{r}}=-\frac{1}{\mu z}-2 \frac{\varepsilon}{n}$,

where two terms represent the collisional and Ohmic energy losses respectively. Coefficient $A(s)=B_{0} / B(s)$ takes into account the variation in the magnetic tube cross-section.

\section{The summary approximation method}

We combine the relative derivatives and rewrite the FokkerPlanck equation of Eq. (10) in the following form:

$$
\begin{aligned}
\frac{\partial f}{\partial \tau}= & -n \sqrt{z} \mu \frac{\partial f}{\partial s}+\left(2 \varepsilon \mu \sqrt{z}+n \frac{1}{\sqrt{z}}\right) \frac{\partial f}{\partial z} \\
& +\left(\varepsilon \frac{1-\mu^{2}}{\sqrt{z}}-n \frac{\mu}{z^{3 / 2}}+n \frac{\left(1-\mu^{2}\right) \sqrt{z}}{2} \alpha_{\mathrm{B}}\right) \frac{\partial f}{\partial \mu} \\
& +n \frac{1-\mu^{2}}{2 z^{3 / 2}} \frac{\partial^{2} f}{\partial \mu^{2}}=\phi_{s} \frac{\partial f}{\partial s}+\phi_{z} \frac{\partial f}{\partial z}+\phi_{\mu} \frac{\partial f}{\partial \mu}+\phi_{2 \mu} \frac{\partial^{2} f}{\partial \mu^{2}} .
\end{aligned}
$$

Equation (28) is solved numerically by using the summary approximation method (Samarskii 2001). This method allows us to study the time dependent Fokker-Planck equation and differs from the one used by Zharkova \& Gordovskyy (2005b) to solve the stationary problem. According to the summary approximation method, the four-dimensional problem is reduced to a chain of three two-dimensional problems. This is achieved by considering the three-dimensional differential operator on the righthand side of Eq. (10) as a sum of one-dimensional operators, each acting on the distribution function separately during one third of the time step. At each time substep, the distribution function is calculated implicitly, hence, the numerical scheme is

$$
\begin{aligned}
& f^{\tau+\frac{1}{3} \Delta \tau}-f^{\tau}=\Delta \tau \phi_{s} L_{S} f^{\tau+\frac{1}{3} \Delta \tau}, \\
& f^{\tau+\frac{2}{3} \Delta \tau}-f^{\tau+\frac{1}{3} \Delta \tau}=\Delta \tau \phi_{z} L_{z} f^{\tau+\frac{2}{3} \Delta \tau}, \\
& f^{\tau+\Delta \tau}-f^{\tau+\frac{2}{3} \Delta \tau}=\Delta \tau\left(\phi_{\mu} L_{\mu}+\phi_{2 \mu} L_{2 \mu}\right) f^{\tau+\Delta \tau},
\end{aligned}
$$

where $L_{\alpha}$ are the finite difference operators that approximate the first order differential operators $\partial / \partial \alpha$. If the coefficient $\phi_{\alpha}$ is positive then the right difference scheme is used, i.e., $L_{\alpha} f=$ $\left(f^{\alpha+\Delta \alpha}-f^{\alpha}\right) / \Delta \alpha$, otherwise the left scheme is used, i.e., $L_{\alpha} f=$ $\left(f^{\alpha}-f^{\alpha-\Delta \alpha}\right) / \Delta \alpha$. The $L_{2 \mu} f=\left(f^{\mu+\Delta \mu}-2 f^{\mu}+f^{\mu-\Delta \mu}\right) / \Delta \alpha^{2}$ is the central difference that approximates the second order derivative $\partial^{2} f / \partial \mu^{2}$. The computational grid has 200 nodes in the $s$ dimension, 50 nodes in the $z$ dimension, and 30 nodes in the $\mu$ dimension. The nodes are distributed logarithmically in the $s$ and $z$ dimensions, and linearly in the $\mu$ dimension.

Equation (29) together with boundary conditions forms a set of linear equations, which, after being solved, infers $f^{\tau+\frac{1}{3} \Delta \tau}$ from known $f^{\tau}$. The distribution function $f^{\tau+\frac{1}{3} \Delta \tau}$ is then used in Eq. (30) to obtain $f^{\tau+\frac{2}{3} \Delta \tau}$. Finally, from Eq. (31) we obtain $f^{\tau+\Delta \tau}$, which is, in turn, used in Eq. (29) in the next step.
Electric field $\varepsilon$ is calculated for each time step according to Eq. (12), where the distribution function is taken from the previous step. Thus, the numerical scheme is not fully implicit. This means that to avoid numerical instability, the time step, $\Delta \tau$, must be shorter than some critical value $\Delta \tau_{\mathrm{c}}$. In practice, the time step was determined by the trial-and-error method. For example, for the energy flux $10^{10} \mathrm{erg} \mathrm{cm}^{-2} \mathrm{~s}^{-1}$ the time step is $1.7 \times 10^{-4} \mathrm{~s}$. It was found that when the energy flux is increased, the time step had to decrease proportionally to keep the numerical scheme stable.

\section{Stationary injection}

We first recapture the major simulation results for the case of a stationary (steady) injection, which helps us to understand the physical process of energy losses by beam electrons during their precipitation. In Zharkova et al. (2009), we studied hard X-ray emission and polarisation produced by a steady beam, while in the current paper we focus more on comparing electron precipitation results obtained for different models of magnetic convergence and on the energy deposition at various depths and times by electron beams with different parameters.

The electron injection starts at $t=0$ and the simulation continues until the stationary state is reached. The initial spectral index of the beam is chosen to be $\gamma=3$, the energy flux at the top boundary is $F_{\text {top }}=10^{10} \mathrm{erg} \mathrm{cm}^{-2} \mathrm{~s}^{-1}$, and the initial angle dispersion is $\Delta \mu=0.2$.

\subsection{Relaxation time to a steady injection}

We first show how the system relaxes to the stationary state. Figure 2 shows the profiles of the electric field and beam density at different times. It can be seen that the electric field relaxes more rapidly than the density. The relaxation time $t_{\mathrm{r}}$, after which the system becomes stationary, can be estimated to be $\sim 0.07 \mathrm{~s}$. As stated in Sect. 2.1, the self-induced electric field is calculated based on the assumption that the beam current changes on timescales longer than the plasma collisional time. Since the collisional time in the corona is about $0.05 \mathrm{~s}$ and decreases with depth, we may conclude that this assumption is correct.

It was found that this relaxation time is longer for a stronger beam, which is the result of a lower ambient plasma density (Fig. 1) and, hence, a larger linear depth for the same column depth. For example, the depth $10^{20} \mathrm{~cm}^{-2}$ corresponds to $2 \times 10^{8} \mathrm{~cm}$ and $11 \times 10^{8} \mathrm{~cm}$ for the beam energy fluxes $10^{10} \mathrm{erg} \mathrm{cm}^{-2} \mathrm{~s}^{-1}$ and $10^{12} \mathrm{erg} \mathrm{cm}^{-2} \mathrm{~s}^{-1}$, respectively. This leads to a longer relaxation time for the atmosphere preheated by a stronger beam, e.g., for the beam energy flux of $10^{12} \mathrm{erg} \mathrm{cm}^{-2} \mathrm{~s}^{-1}$, the relaxation time is found to be $\sim 0.2 \mathrm{~s}$.

\subsection{Effects of collisions and electric field $\left(\alpha_{B}=0\right)$}

The local maximum, which appears in the density profile for a depth of about $\sim 2 \times 10^{19} \mathrm{~cm}^{-2}$ (Fig. 2a), is caused by beam deceleration, while the flux of electrons remains nearly constant. After this depth, most of the electrons leave the distribution (thermalise) by reducing their energy below the energy $z_{\min }$, and the density rapidly decreases at a depth of about $\sim 5 \times 10^{19} \mathrm{~cm}^{-2}$ (Fig. 2a). This corresponds to the stopping depth for electrons with energies close to the lower cut-off energy $(12 \mathrm{keV})$. The electrons with higher energies can travel deeper (see Fig. 3). In particular, it can be seen that electrons with energies $>500 \mathrm{keV}$ can travel down to the photosphere almost without any energy 


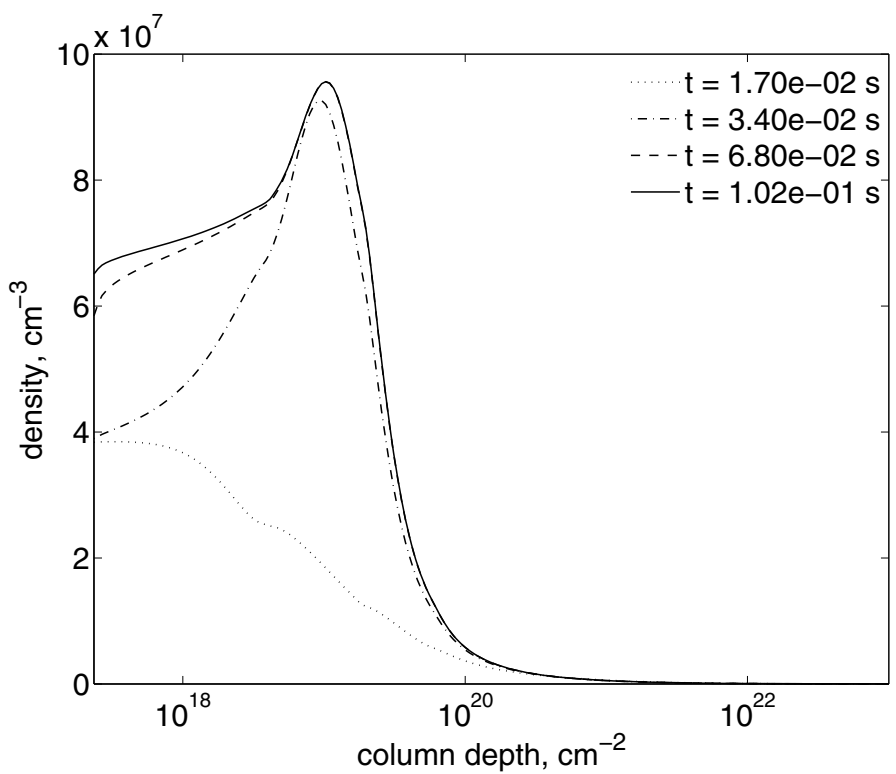

(a)

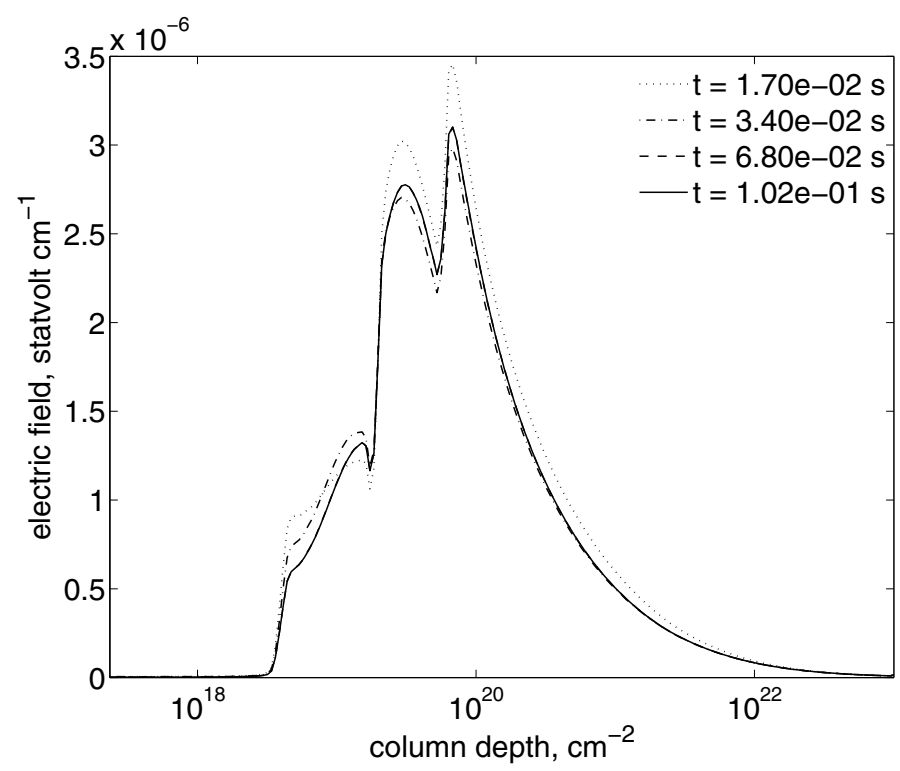

(b)

Fig. 2. Electron density a) and self-induced electric field b) profiles, where $t$ is time passed after the injection is "turned on". Collisions and electric field are taken into account. The beam parameters (see Eq. (15)) are $\gamma=3, F_{\text {top }}=10^{10} \mathrm{erg} \mathrm{cm}^{-2} \mathrm{~s}^{-1}$ and $\Delta \mu=0.2$.

losses. Figure 3 confirms the results obtained by Zharkova \& Gordovskyy (2006, see their Table 1).

The depth profile of the electric field (Fig. 2b) has some noticeable variations at column depths of about $4 \times 10^{18} \mathrm{~cm}^{-2}$, $2 \times 10^{19} \mathrm{~cm}^{-2}$, and $6 \times 10^{19} \mathrm{~cm}^{-2}$. These features are located at the same depths where the temperature profile (Fig. 1) exhibits sharp decreases leading to the sharp decreases in plasma conductivity given by Eq. (13). However, the electron density fluctuation associated (through the Gauss's Law) with this electric field profile is found to be of the order of $10^{-4} \mathrm{~cm}^{-3}$ and can be neglected.

In Fig. 4, we plot the differential flux spectra at different depths for the forward $(\mu>0)$ and backward $(\mu<0)$ moving electrons. It can be seen that the self-induced electric field

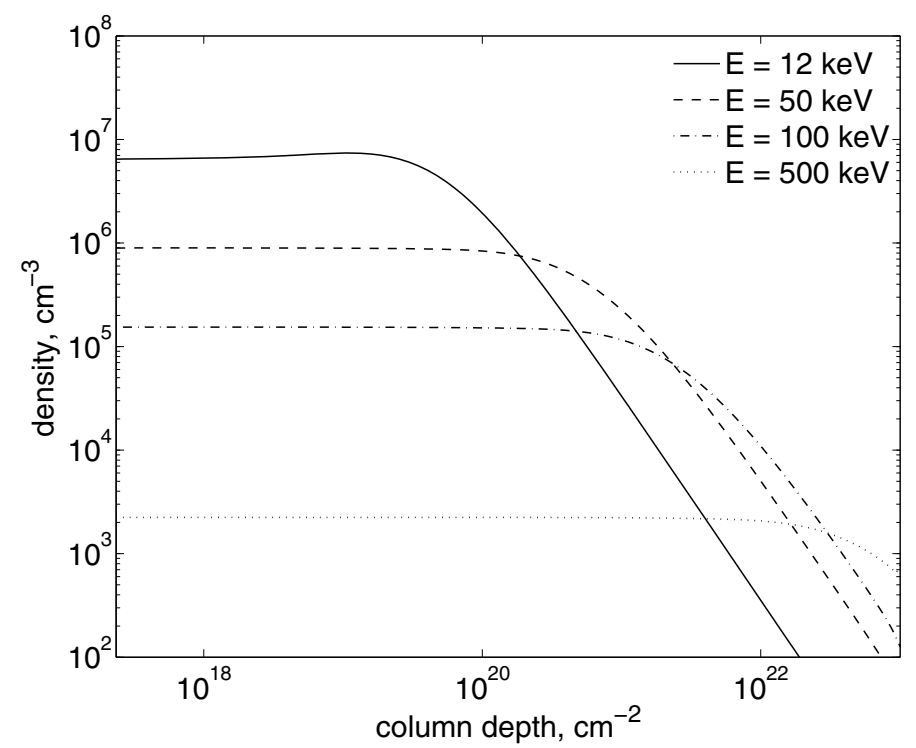

Fig. 3. Beam electron density in different energy bands, if only collisions are taken into account. Beam parameters are the same as in Fig. 2.

does not change the spectra of the downward $(\mu>0)$ moving electrons but affects only the spectra of the upward $(\mu<0)$ moving ones. Since the pitch angle diffusion due to the electric field is more effective for lower energy electrons, the spectra of the returned electrons is enhanced at low and mid energy (Fig. 4d).

The number of electrons returning back to the source plotted in Fig. 5a is lower than when the self-induced electric field is not taken into account. However, even without the electric field $\varepsilon$, a number of electrons with $\mu<0$ is essential owing to the pitch angle scattering (second and third terms on the right hand side of Eq. (10)).

The heating function plot (Fig. 5b) shows that if a selfinduced electric field is taken into account the heating is more effective at an upper column depth $\left(2 \times 10^{19} \mathrm{~cm}^{-2}\right)$ than a much lower one $\left(10^{20} \mathrm{~cm}^{-2}\right)$ in the pure collisional beam relaxation, which is consistent with the results obtained by Emslie (1980). Indeed, the inclusion of the electric field decreases the stopping depth (Zharkova \& Gordovskyy 2006) and increases the number of returning electrons, thus, reducing the number of electrons at larger depths. All these factors lead to an upward shift in the heating function maximum. The theoretical heating curve for pure collisions (Syrovatskii \& Shmeleva 1972) is plotted in Fig. 5b.

\subsection{Effects of a magnetic convergence}

The converging magnetic field acts as a magnetic mirror and can increase the number of the electrons that move upwards. We determine how large the magnetic convergence parameter, $\alpha_{B}$, should be to have any noticeable effect on the distribution of beam electrons. To do so, we compare the terms in front of the $\partial f / \partial \mu$ symbol in Eq. (28). Since the collisional pitch angle diffusion is much lower than the one caused by the electric field (see Fig. 5a), we compare the effects of the magnetic convergence with those caused by the electric field. The magnetic convergence effects are stronger if $\alpha_{B}>2 \varepsilon /(n z)$. To estimate this expression, we plot the dimensionless ratio $\varepsilon / n$ in Fig. 6 . The minimal value of the ratio $\varepsilon / n$ in the interval from $s=s_{\min }$ to the stopping depth of low energy $(z=1)$ electrons, $\sim 5 \times 10^{19} \mathrm{~cm}^{-2}$, is about 0.2 . Thus, the magnetic convergence would be more 


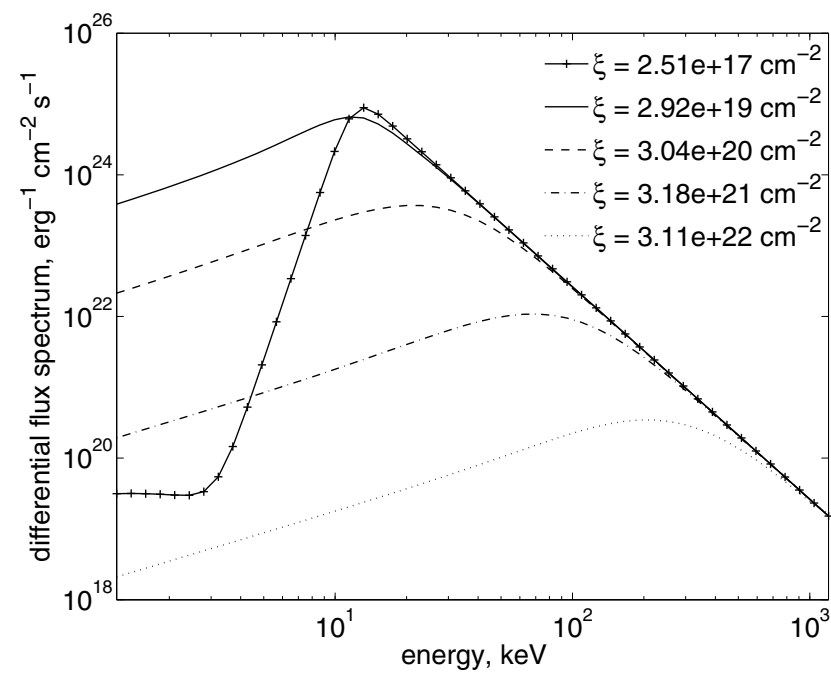

(a) collisions, $\mu>0$

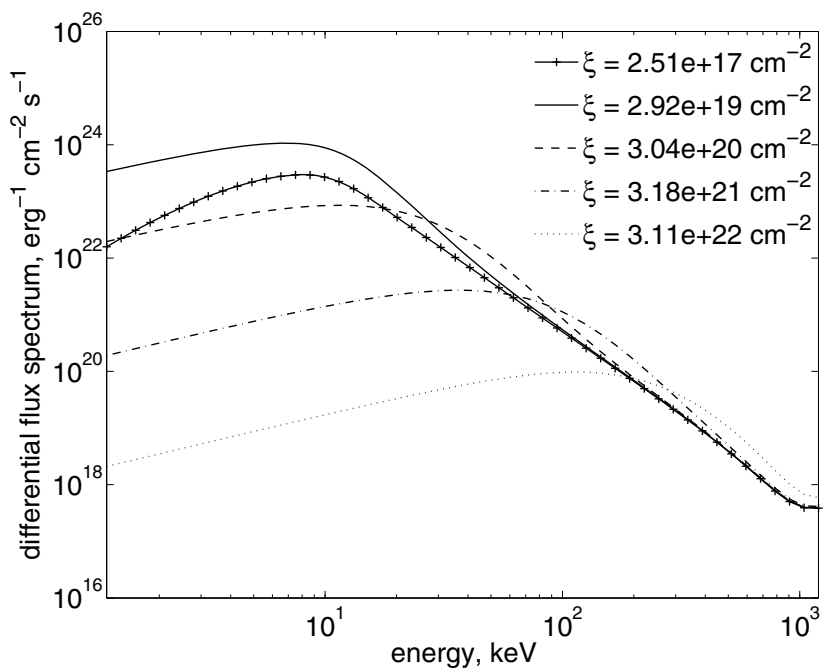

(c) collisions, $\mu<0$

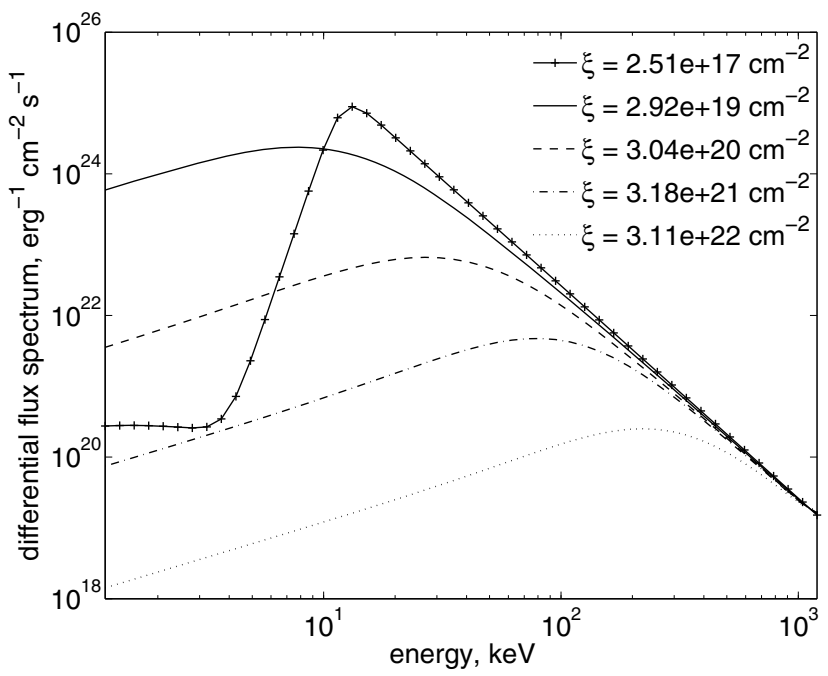

(b) collisions and electric field, $\mu>0$

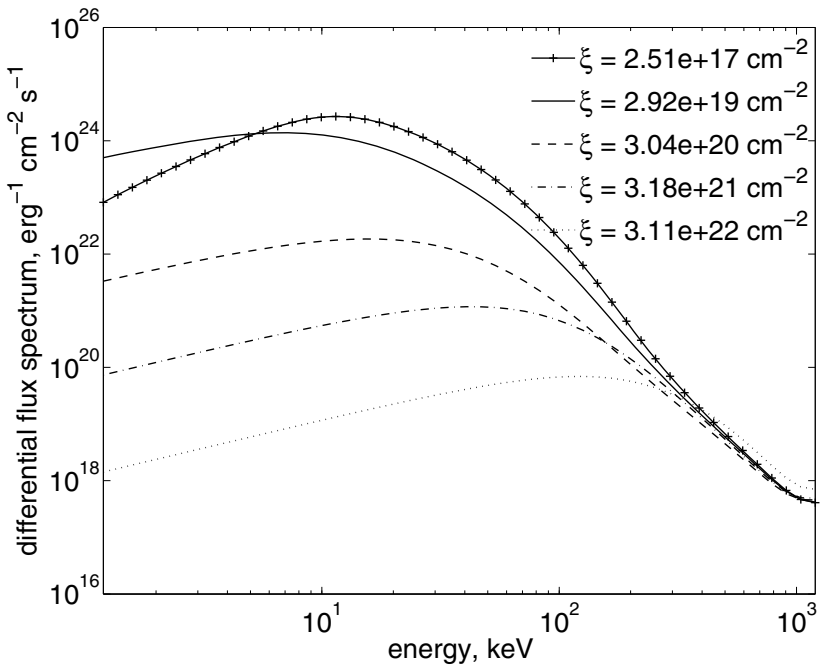

(d) collisions and electric field, $\mu<0$

Fig. 4. Differential flux spectra of the beam electrons integrated over the positive and negative pitch angles. Beam parameters are the same as in Fig. 2.

effective than the electric field for the electrons with energies higher than the cut-off energy if $\alpha_{B} \gtrsim 0.4$. High energy electrons can travel much deeper into the chromosphere (see Fig. 3), where the ratio $\varepsilon / n$ can be as low as $2 \times 10^{-4}$, thus the magnetic convergence would be more effective for them if $\alpha_{B} \gtrsim 4 \times 10^{-6}$.

In the following subsections, we present the results of simulations for different models of the converging magnetic field. These results are illustrated by the mean flux spectra plots (Fig. 7a) for the upward $(\mu<0)$ moving electrons, while the spectra of the downward moving electrons are found to be very close for all convergence models. The energy deposition profiles for different magnetic field approximations are shown in Fig. $7 \mathrm{~b}$.

\subsubsection{Exponential approximation of a magnetic field convergence}

Following the approximation proposed by Leach \& Petrosian (1981), we assume that the convergence parameter does not depend on depth

$\alpha_{B}=\alpha_{B 0}=$ const., and that then the magnetic field variation is given by

$B(s)=B_{0} \exp \left(\alpha_{B 0}\left(s-s_{\min }\right)\right)$.

We assume that the magnetic field at the depth $s_{\max }$ is 1000 times stronger than at the depth $s_{\min }$, which then implies that $\alpha_{B} \approx$ $\ln (1000) / s_{\max }=7.5 \times 10^{-3}$. As we discussed earlier, the effect of the magnetic convergence with such a low value of $\alpha_{B}$ would be noticeable only for high energy $(>100 \mathrm{keV})$ electrons. This is clearly illustrated by a comparison of the mean flux spectra of the moving upward electrons and heating function obtained with and without magnetic convergence (see solid and dashed curves in Fig. 7). While the electric field returns mostly the low and mid energy electrons and makes the spectrum of the returning electrons softer (in comparison with the purely collisional case), the magnetic mirror returns back the high energy electrons and makes their spectrum harder and similar to the initial power law. On the other hand, magnetic convergence reduces the heating at the deeper layers (Fig. 7b), where it is caused by high energy electrons, because they were mirrored back to the corona. 


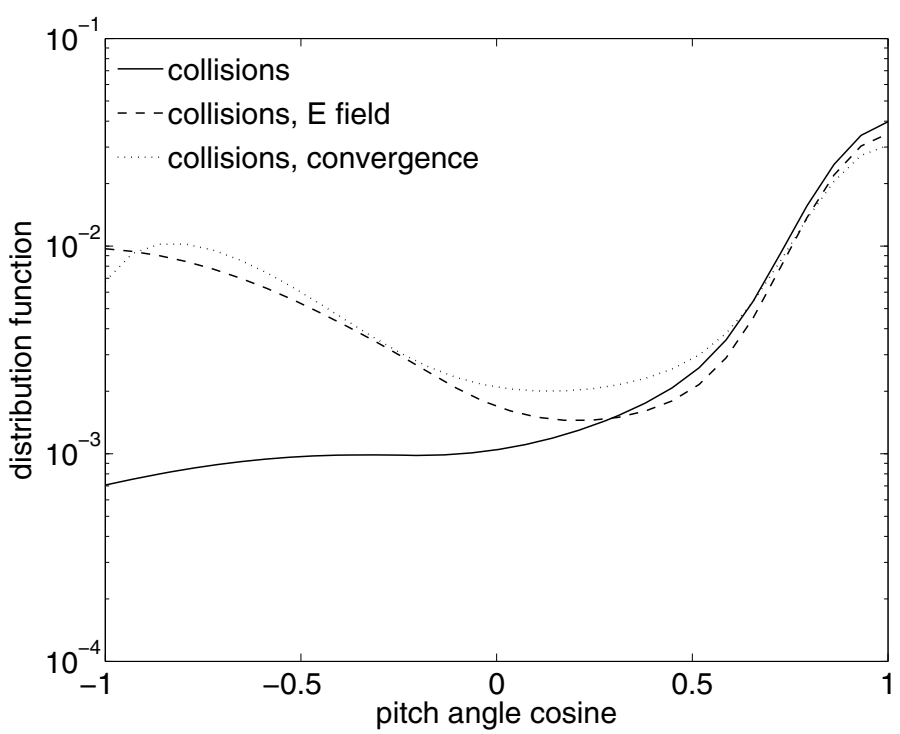

(a)

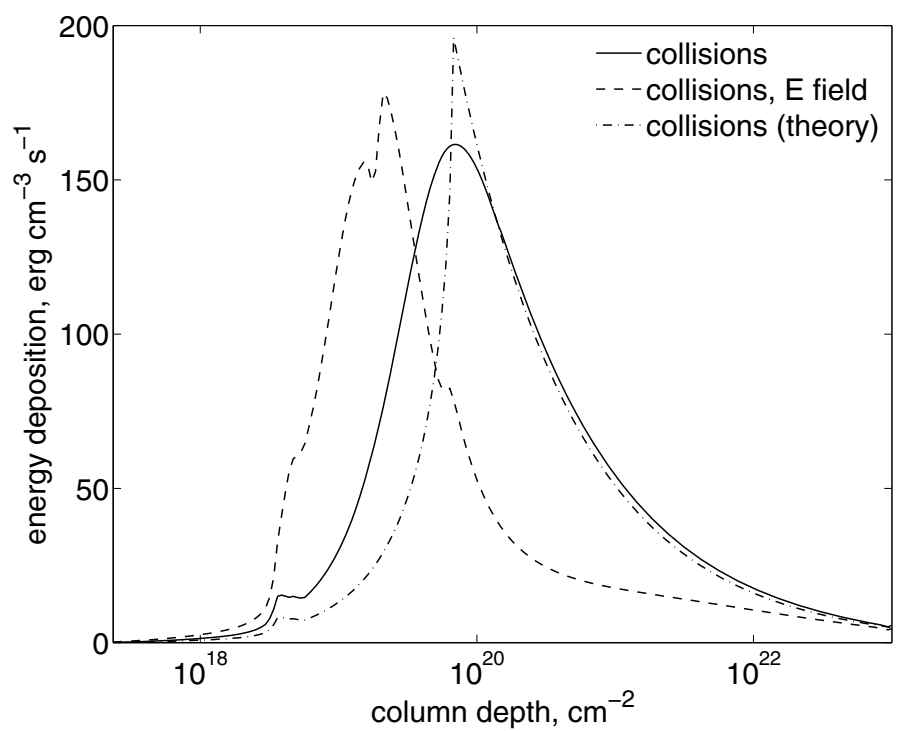

(b)

Fig. 5. Pitch angle distribution a) and heating function of the beam b). Beam parameters are the same as in Fig. 2 and the magnetic convergence (for dotted curve) is given by Eq. (36).

\subsubsection{Parabolic approximation}

McClements (1992) suggested the following profile of a magnetic field variation:

$B(s)=B_{0}\left(1+\frac{\left(s-s_{\min }\right)^{2}}{s_{0}^{2}}\right)$.

If $B\left(s_{\max }\right) / B\left(s_{\min }\right)=1000$ then $s_{0} \approx s_{\max } / \sqrt{(1000)}=31.6$. The convergence parameter is

$\alpha_{B}(s)=\frac{2\left(s-s_{\min }\right)}{s_{0}^{2}+\left(s-s_{\min }\right)^{2}}$.

The magnetic convergence parameter $\alpha_{B}$ at maximum is $1 / s_{0} \approx$ $3.16 \times 10^{-2}$. This value is not high enough to affect all the beam electrons, but as is seen in Fig. 7, this convergence model, in a similar way to the previous one, increases the number of high energy electrons with negative $\mu$ and reduces the heating of deep

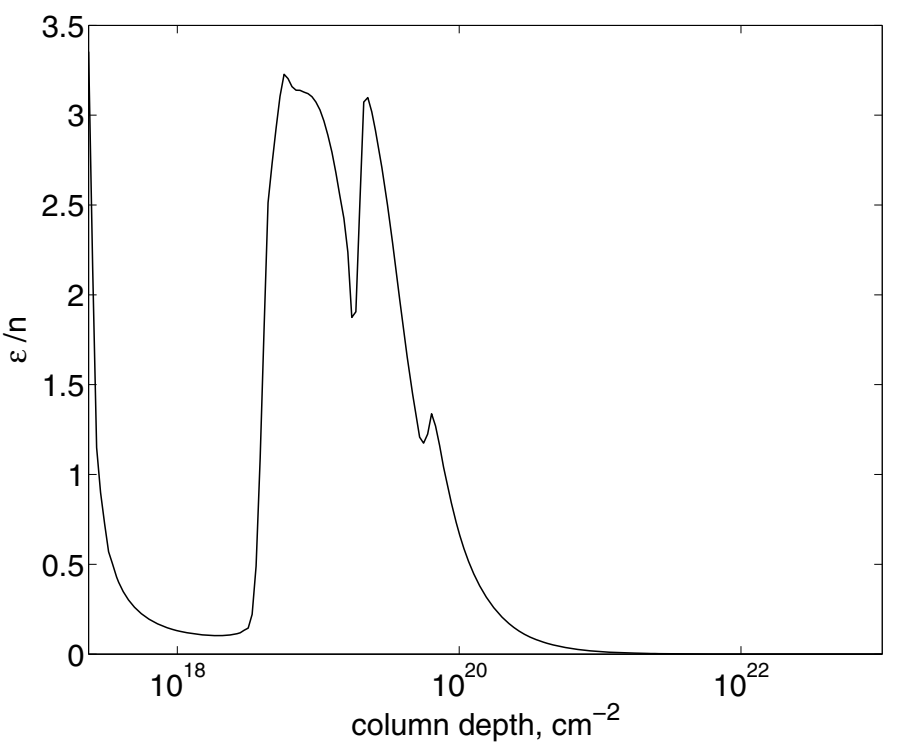

Fig. 6. The ratio of the dimensionless electric field to the ambient plasma density as a function of column depth. Collisions and electric field are taken into account. Beam parameters are the same as in Fig. 2.

atmosphere layers. We note, also, that McClements (1992) considered a constant plasma density, and in their model, the profile of the magnetic field given by Eq. (34) can be more effective, while in our case plasma density exponentially increases with depth. Here we assumed that the magnetic field changes according to Eq. (34) for the range of column depths. However, it would be more appropriate to assume that the magnetic field variation is different in the corona and chromosphere. This approach is discussed in the following section.

\subsubsection{Hybrid approximation of magnetic field}

In this model, we propose that $\alpha_{\mathrm{B}}$ is almost constant at small depth (in the corona) and tends to zero after some depth $s_{0}$ (in the chromosphere)

$\alpha_{B}(s)=\alpha_{B 0} \frac{s_{0}^{2}}{s_{0}^{2}+\left(s-s_{\min }\right)^{2}}$,

and the magnetic field variation is then

$$
\begin{aligned}
B(s) & =B_{0} \exp \left(\int_{s_{\min }}^{s} \alpha\left(s^{\prime}\right) \mathrm{d} s^{\prime}\right) \\
& =B_{0} \exp \left(\alpha_{\mathrm{B} 0} s_{0} \arctan \left(\frac{s-s_{\min }}{s_{0}}\right)\right) .
\end{aligned}
$$

At shallow depths, where $s \ll s_{0}$, the magnetic field varies as $B \approx B_{0} \exp \left(\alpha_{B 0}\left(s-s_{\min }\right)\right)$, and at deeper layers, $s \gg s_{0}$, the magnetic field is constant, $B \approx B_{0} \exp \left(\alpha_{B 0} s_{0} \pi / 2\right)$. In most of the simulations (where not stated explicitly), we accept $\alpha_{B 0}=10$ and $s_{0}=0.2$ (or $2.2 \times 10^{19} \mathrm{~cm}^{-2}$, corresponding to the transitional region), which implies that the ratio $B\left(s_{\max }\right) / B\left(s_{\min }\right)$ equals 23.1 .

Electrons with velocities inside the loss-cone are not reflected by the magnetic mirror and reach deeper layers. For the current convergence model, the critical pitch angle cosine of the loss-cone is $\mu_{\mathrm{lc}}=\sqrt{1-B\left(s_{\min }\right) / B\left(s_{\max }\right)} \approx 0.98$. This means that for the accepted initial angle dispersion of 0.2 , about $90 \%$ of the electrons are reflected back. 


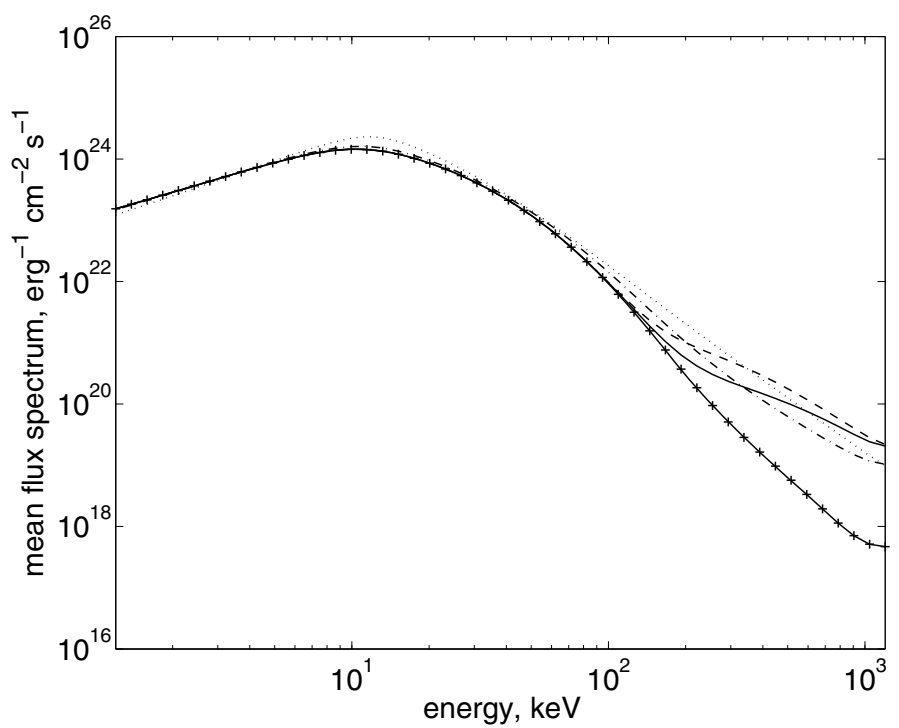

(a)

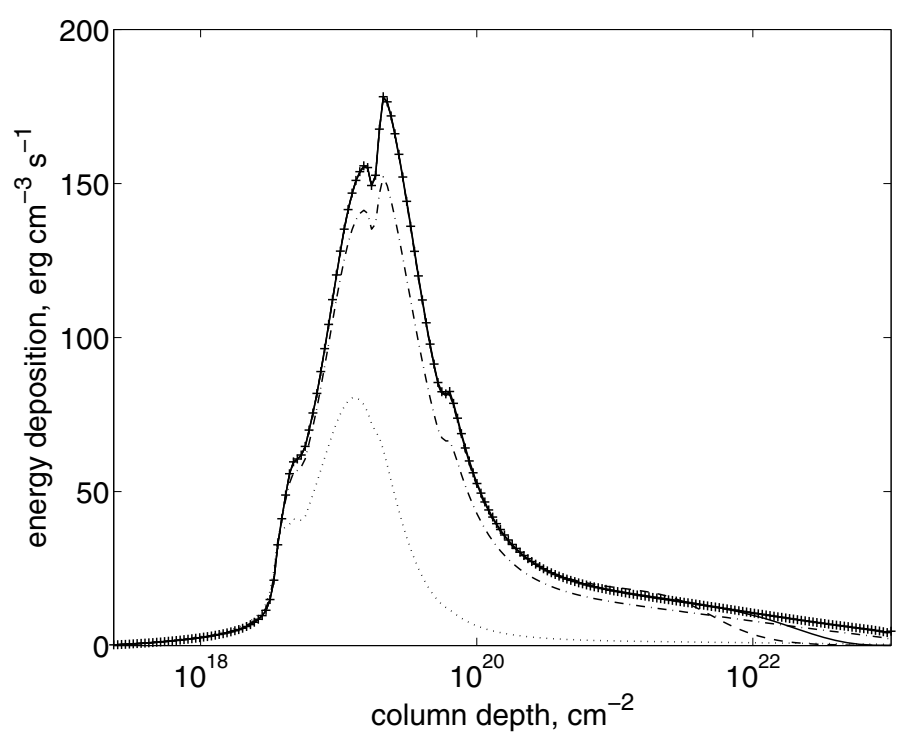

(b)

Fig. 7. Mean flux spectra a) of the upward $(\mu<0)$ propagating electrons and energy deposition b) without (crosses) and with magnetic convergence given by Eq. (33) (solid curve), Eq. (34) (dashed curve), Eq. (37) (dotted curve), and Eq. (39) (dot-dashed curve). Beam parameters are the same as in Fig. 2.

As can be seen in Fig. 5b, the effect of magnetic convergence on the pitch angle distribution is similar to the effect of the electric field. However, since the electric field is most effective for the electrons with $\mu= \pm 1$, the pitch angle distribution reaches a maximum at $\mu=1$ when convergence is not taken into account. In contrast, the magnetic field does not affect electrons moving along the field lines, thus, the angle distribution of the upward moving electrons has a maximum at $\mu_{\mathrm{m}} \approx-0.8$ (Fig. 5a), which is consistent with the conclusions of Zharkova \& Gordovskyy (2006).

Since the convergence parameter $\alpha_{\mathrm{B}}$ is relatively high in this model, the entire spectrum of electron energy is affected (see Fig. 7a). The energy deposition profile for this magnetic field approximation (Fig. 7b) indicates that the heating is only about $30 \%$ of the heating produced in the case of constant magnetic

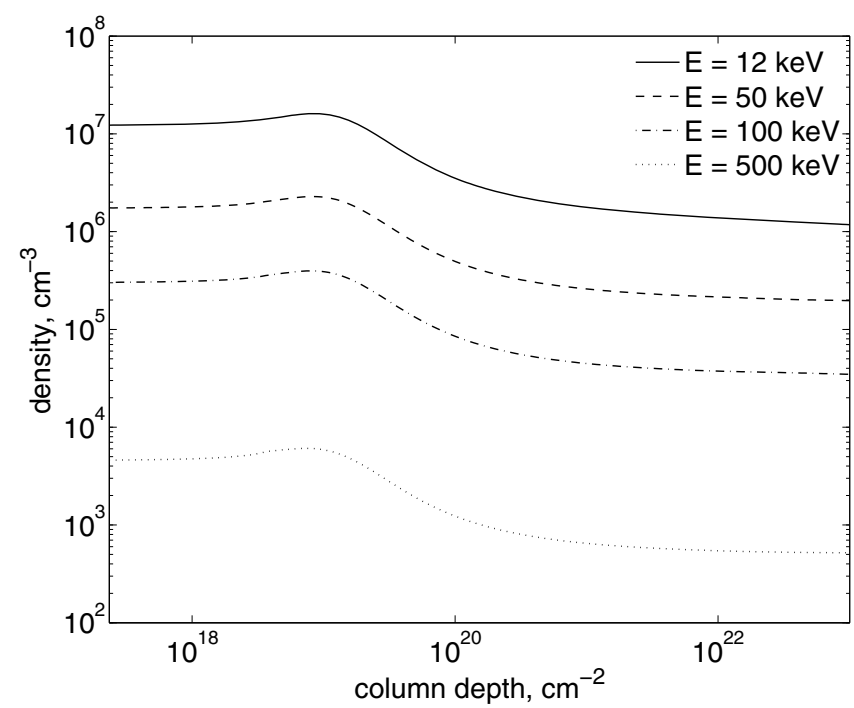

(a) convergence

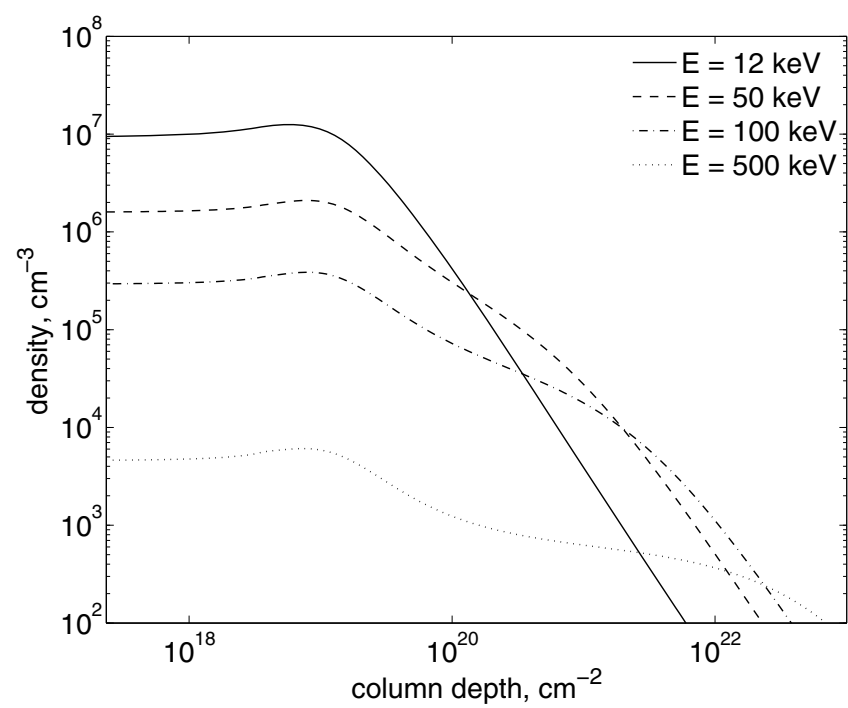

(b) convergence and collisions

Fig. 8. Beam electron density in different energy bands. The magnetic convergence parameter is given by Eq. (36). Beam parameters are the same as in Fig. 2.

field, which is because many electrons are reflected by the magnetic mirror before they enter the dense plasma.

The profiles of electron density for different energies are plotted in Fig. 8. If only magnetic convergence is taken into account (Fig. 8a), it can be seen that magnetic mirroring does not depend on the electron energy. Electrons whose pitch angles are outside the loss-cone are turned back at a depth $\sim 10^{19}-10^{20} \mathrm{~cm}^{-2}$. The remaining electrons (the pitch angles of which are inside the loss-cone) can travel down to the lower boundary of the atmosphere. When collisions are taken into account, electrons, especially those with low energies, lose their energy due to collisions (Fig. 8b). It is important to compare collisional beam relaxation with and without magnetic convergence as plotted in Figs. 8b and 3, respectively. It is noticeable that the combination of the effects of collisions and convergence is stronger than the sum of the two separate effects. This occurs because electrons with the initial pitch angles inside the losscone are scattered by collisions to pitch angles that fall out of 


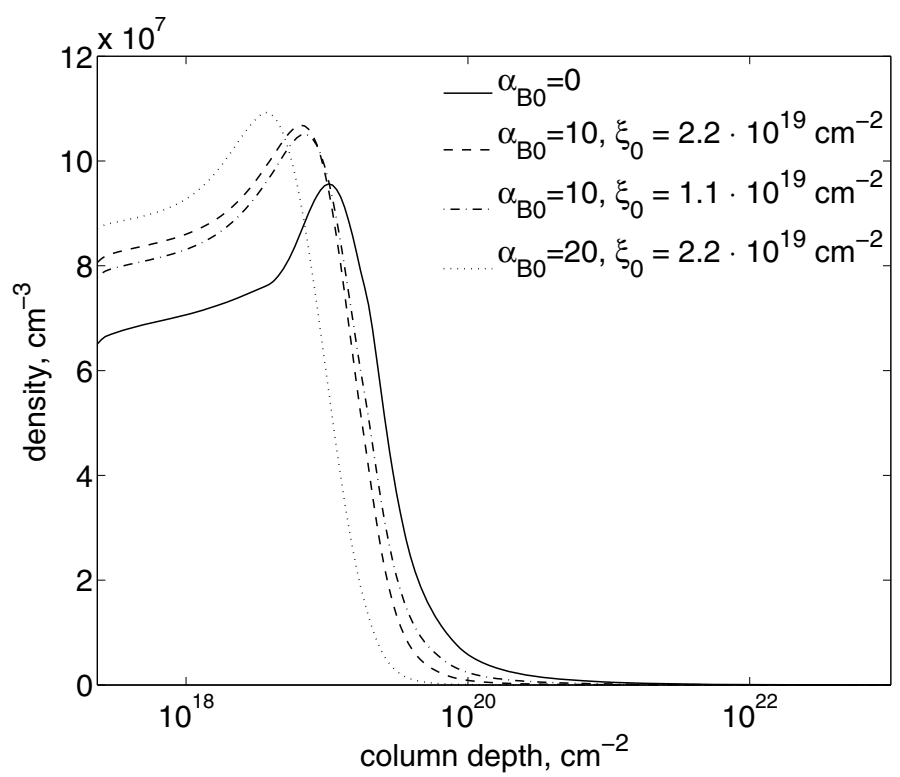

(a)

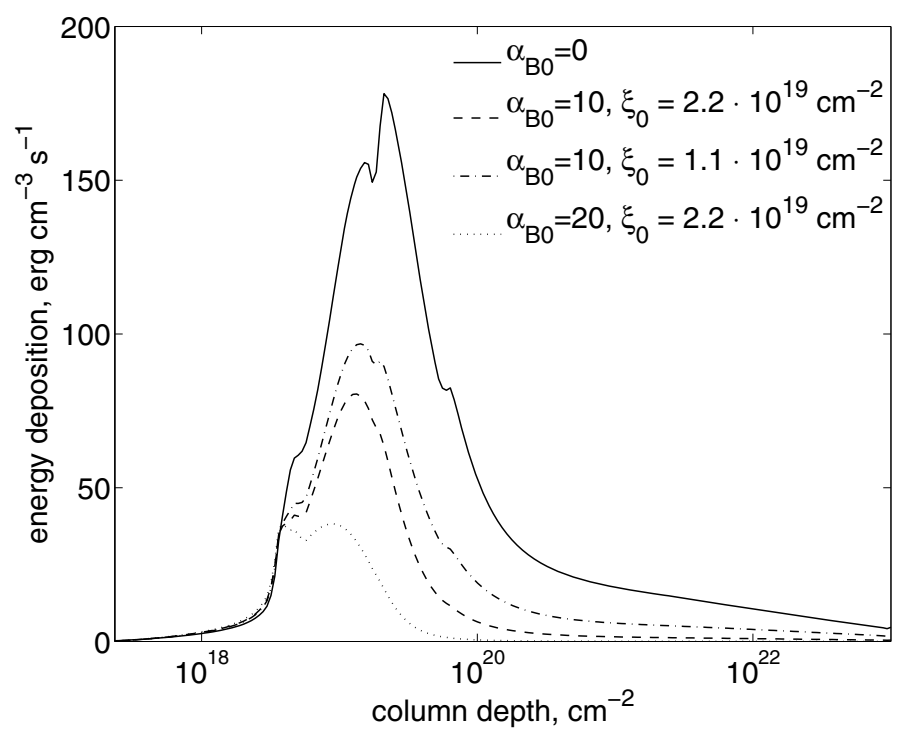

(b)

Fig. 9. Beam density a) and energy deposition b) as a function of column depth. The magnetic convergence parameter is given by Eq. (36) with various $\alpha_{B 0}$ and $s_{0}$. Beam parameters are the same as in Fig. 2.

the loss-cone, therefore, more electrons are returned back by the magnetic mirror.

In Fig. 9, the results of simulations are presented for different magnitudes for the parameters $\alpha_{B 0}$ and $s_{0}$ of the magnetic convergence model given by Eq. (36). The increase in $\alpha_{B 0}$ clearly affects the beam electrons by reducing the depth of their penetration and by increasing the number of returning electrons. It is obvious that the system would be sensitive to the variation in $s_{0}$, if it is smaller than the penetration (stopping) depth. This is proven in Fig. 9a.

\subsubsection{Magnetic field model fitted to the observations}

Although the magnetic field cannot be directly measured in the solar atmosphere, there are some indirect techniques that allow the magnitude of the magnetic field to be estimated. The coronal magnetic field can be determined from radio observations of gyro-resonance emission (Lang et al. 1993; Brosius \& White 2006). In particular, Brosius \& White (2006) suggest that the magnetic scale height above sunspots, $L_{B \text { cor }}=B / \Delta B$, is $\sim 7 \mathrm{Mm}$. On the other hand, Kontar et al. (2008) determined the chromospheric magnetic field by measuring the sizes and heights of hard X-ray sources in different energy bands. They found that the chromospheric magnetic scale height, $L_{B \mathrm{chr}}$, is $\sim 0.3 \mathrm{Mm}$. Assuming that the magnetic scale height, $L_{B}$, changes linearly with depth $l$, the convergence parameter is

$\alpha_{B}(s)=\frac{\alpha_{B 0}}{n L_{B}}$,

where $\alpha_{B 0}=E_{0}^{2} /\left(\pi e^{4} n_{0} \ln \Lambda L_{B \text { cor }}\right)=15.7$, and the dimensionless magnetic scale height as a function of the column depth is

$L_{B}=1-\left(1-\frac{L_{B \mathrm{chr}}}{L_{B \mathrm{cor}}}\right) \frac{l(s)}{l\left(s_{\max }\right)}$,

where the linear depth is $l(s) \propto \int n(s)^{-1} \mathrm{~d} s$. Since the magnetic field is defined as a function of the linear depth, the model depends on the density profile $n(s)$ of the background plasma.

As can be seen in Fig. 7a, this magnetic convergence affects electrons of all energies. This leads to about a $20 \%$ reduction in the heating produced by beam electrons in comparison to the constant magnetic field profile (Fig. 7b).

\section{Impulsive injection}

We now consider the precipitation of electron beam injected as a short impulse. The early SMM (Kiplinger et al. 1983) and CORONAS/IRIS (Charikov et al. 2004) observations identify millisecond impulses in the hard X-ray emission from solar flares. Furthermore, the electron acceleration time in a reconnecting current sheet was shown by Siversky \& Zharkova (2009) to be as short as $10^{-5} \mathrm{~s}$. The timescale of a beam of accelerated electrons should therefore be rather short. In this section, we study the evolution in these short impulses in the solar atmosphere. The injection time, $\delta t$, is chosen to be $1.7 \times 10^{-3} \mathrm{~s}$, which is much shorter than the relaxation time $t_{\mathrm{r}} \approx 0.07 \mathrm{~s}$, found in Sect. 4.1 (see Fig. 2). The default parameters of the beam are similar to those for stationary injection: the initial spectral index of the beam is $\gamma=3$, the maximal energy flux at the top boundary is $F_{\text {top }}=10^{10} \mathrm{erg} \mathrm{cm}^{-2} \mathrm{~s}^{-1}$, and the initial angle dispersion is $\Delta \mu=0.2$. The energy deposition profiles produced both by a softer beam $(\gamma=7)$ and by a stronger beam $\left(F_{\text {top }}=10^{12} \mathrm{erg} \mathrm{cm}^{-2} \mathrm{~s}^{-1}\right)$ are also obtained.

The impulse injection, obviously, leads to a lower density of electrons at a given depth in comparison with the stationary injection (see Figs. 2a and 10a). A lower density produces a lower self-induced electric field. Thus, in the case of a short impulsive injection, the electric field does not affect the distributions so much. As a result, the only mechanism that can increase the number of returning electrons is magnetic convergence. As mentioned above in the current study, we assume that the beam current is always compensated by the plasma return current, thus the self-induced electric field develops immediately. However, as shown by van den Oord (1990), the neutralisation time of the beam current is of the order of the collisional time. Thus, the effect of the electric field on short impulses can be even smaller than our estimations.

Anisotropic scattering of beam electrons in collisions with the ambient plasma causes the pitch angle distribution to become 


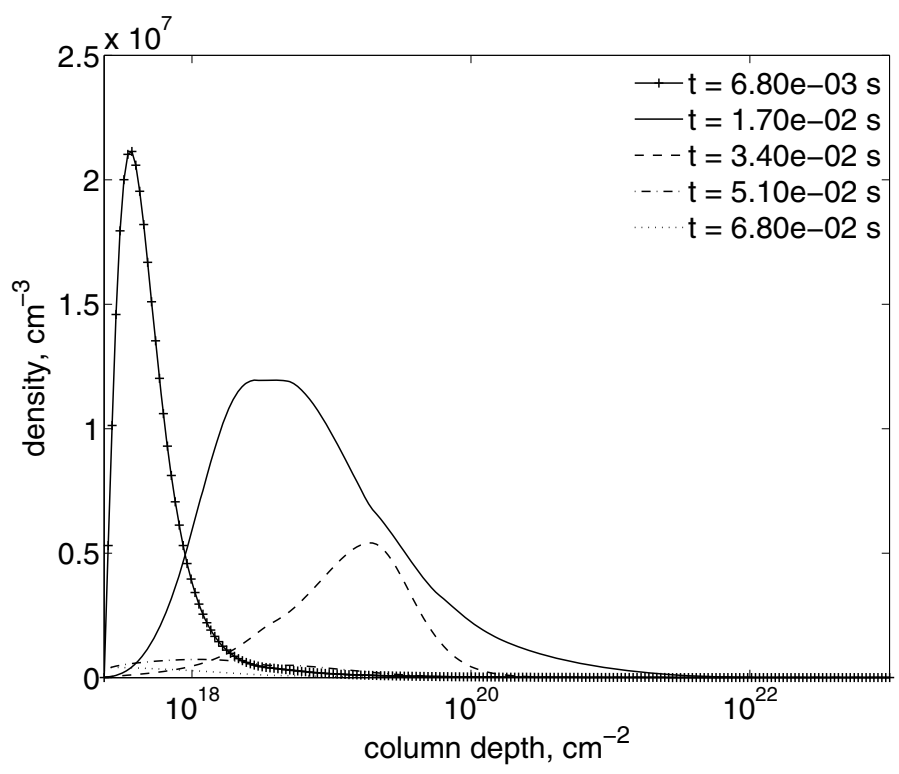

(a)

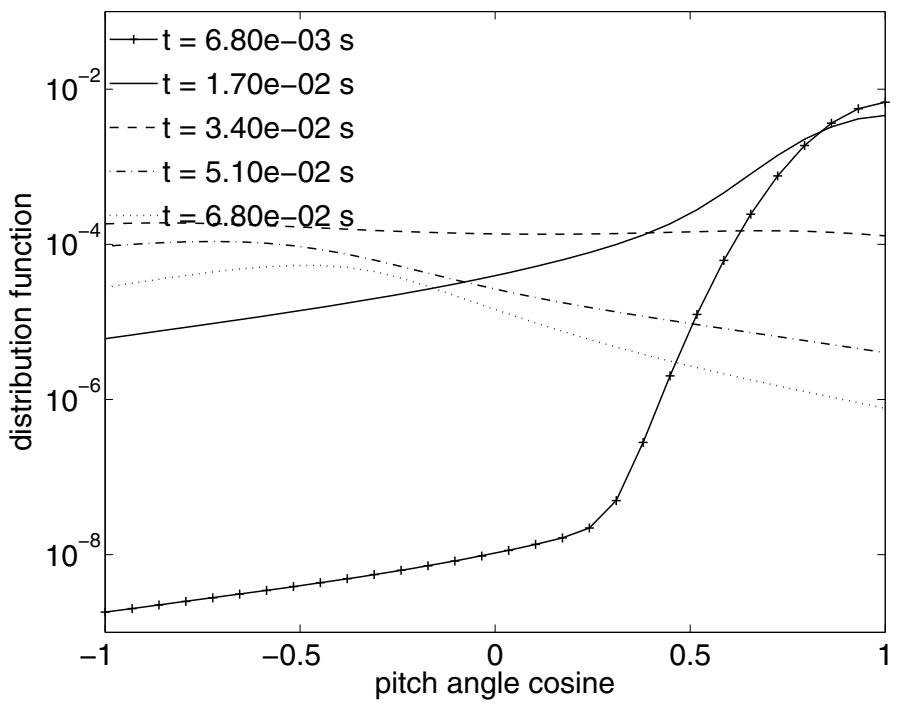

(b)

Fig. 10. Electron density at impulsive injection as a function of column depth a) and pitch angle b). Collisions and the electric field are taken into account. The beam parameters, see Eq. (15), are $\gamma=3, F_{\text {top }}=$ $10^{10} \mathrm{erg} \mathrm{cm}^{-2} \mathrm{~s}^{-1}, \Delta \mu=0.2$, and injection time, $\delta t=1.7 \times 10^{-3} \mathrm{~s}$.

flatter with time (see Fig. 10b). The electrons propagating downward reach depths of high ambient plasma density, lose their energy due to collisions, and leave the distribution (becoming thermalised) when their energy is less than $z_{\min }$. In contrast, the returning electrons move into less dense plasma almost without losing any energy, but gaining energy in the self-induced electric field. Thus, after some time the number of upward moving electrons can exceed the number of downward moving ones, which is clearly seen in Fig. 10b. The angle distributions show that after $\sim 3.4 \times 10^{-2} \mathrm{~s}$, most of the downward propagating electrons are gone and the majority of electrons have $\mu<0$, i.e., they move back to their source in the corona.

\subsection{Energy spectra}

Since the first term in the right-hand side of Eq. (10), which is responsible for the energy losses due to collisions, is proportional to $z^{-1 / 2}$ (where $z$ is the dimensionless energy), one might expect that electron spectra would become harder with time. However, the downward moving electrons of higher energy both reach the dense plasma and lose their energy faster than lower energy electrons, which makes the energy spectra softer with time (Fig. 11a). The same is valid for the spectra of the upward moving electrons. In this case, the high energy electrons escape the distribution faster by reaching the top boundary $\left(s=s_{\min }\right)$. Because of this effect, the power law index can increase from the initial value of 3 , to 4 during the beam evolution (Fig. 11a).

A magnetic field on its own cannot change the energy of the electrons. However, a converging magnetic field acts as a magnetic mirror and returns most of the electrons back to their source. As shown above in Fig. 7a, for the high energy electrons the magnetic convergence is more effective than the electric field and pitch angle diffusion. Thus, high energy electrons can quickly escape through the $s=s_{\min }$ boundary and the power law index can reach higher values than for a constant magnetic field. For example, for the magnetic field profile given by Eq. (37) the power law index increases from the initial value of 3 to 8 (Fig. 11c). If the convergence parameter is defined by Eq. (39), the initial power law distribution converts to some kind of quasithermal distribution with drop at high energies (Fig. 11d).

\subsection{Energy deposition}

Figure 12 shows the evolution in the energy deposition, or heating functions, when different precipitation effects are taken into account. In the purely collisional case (Fig. 12a), the heating maximum appears at the bottom boundary, moves upwards with time and vanishes near column depths of $\sim 10^{19}-10^{20} \mathrm{~cm}^{-2}$. This evolution is consistent with stopping depths obtained for electrons with different energies (Fig. 3). The high energy electrons are the first to reach depths where the density is high enough to thermalise them. Less energetic electrons travel longer because of their lower velocity but lose their energy higher in the atmosphere. Thus, the heating function maximum moves with precipitation time from the photosphere $(10 \mathrm{~ms})$ to the lower (17 ms) and than upper (22 ms) chromosphere towards the stopping depth of the low energy electrons, where it vanishes.

In the presence of a self-induced electric field (Fig. 12b), the heating by collisions becomes smaller than in the purely collisional case because some electrons are reflected by the electric field and do not reach dense plasma and, thus, do not deposit their energy into the ambient plasma. On the other hand, there is a second maximum on the heating function, which is also caused by the energy losses due to Ohmic heating by the self-induced electric field. This maximum does not move but grows in time with more electrons coming into the region with a high electric field (see Fig. 2b).

As shown in Sect. 4.3.3 for the magnetic convergence given by Eq. (37), only about $10 \%$ of the electrons can escape through the loss-cone and heat the deep layers. Thus, magnetic convergence reduces the energy deposition substantially at lower atmospheric levels because of the mirroring of the electrons back to the top, which shifts the heating maximum upwards into the corona (Figs. 12c and d).

The heating function of a beam with the initial spectral index $\gamma=7$ is shown in Fig. 13. In contrast to the $\gamma=3$ beam, the heating peak appears at shallower depths and moves downwards with time. Apparently, this occurs because the number of high energy electrons is extremely low for a softer beam $(\gamma=7)$, and, thus, the heating that they can produce at greater depths is 


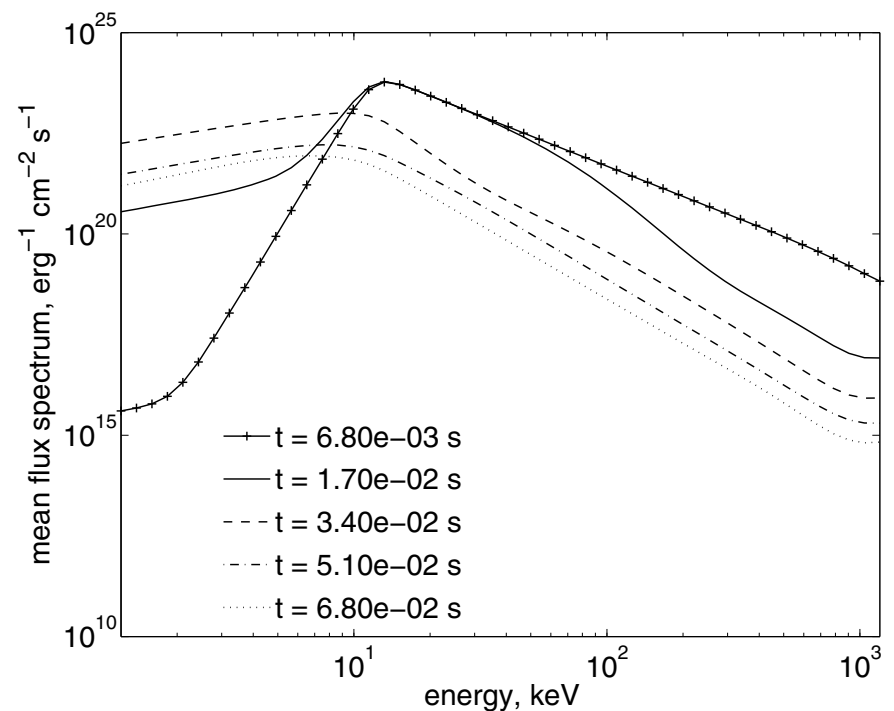

(a) collisions

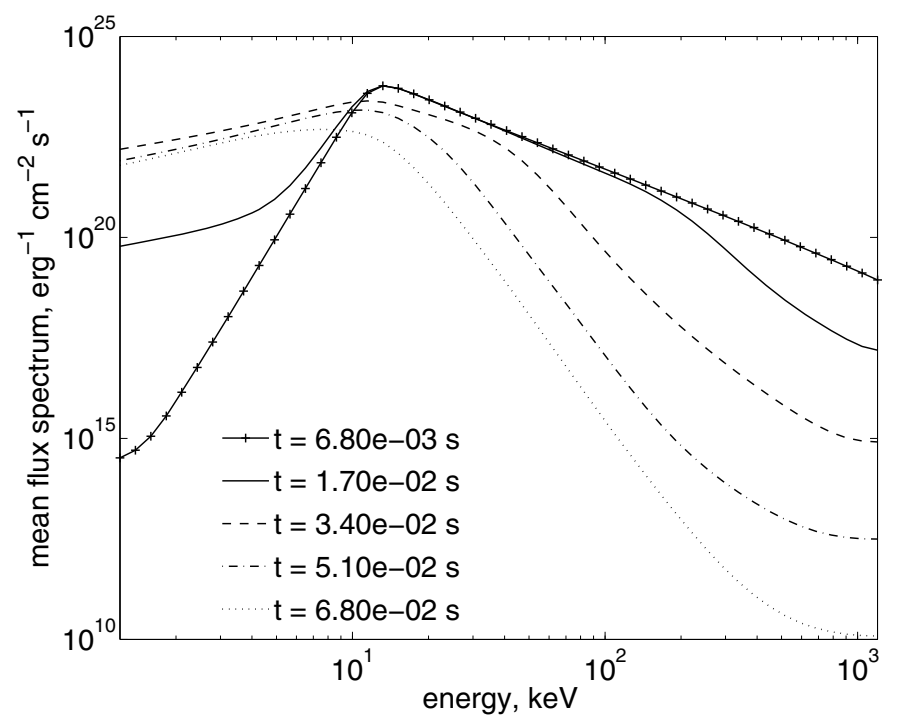

(c) collisions and convergence according to Eq. (36)

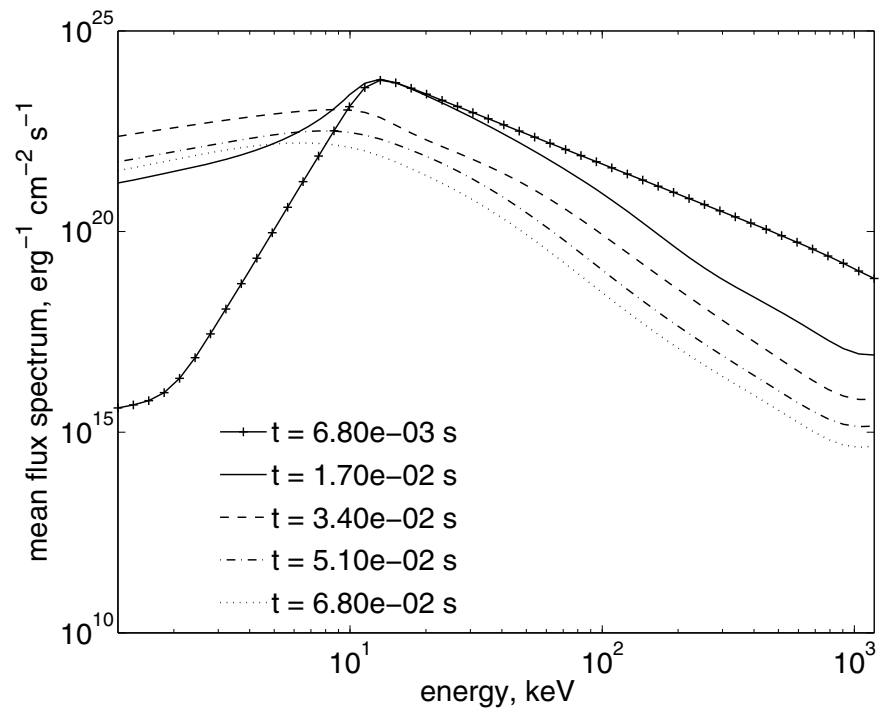

(b) collisions and electric field

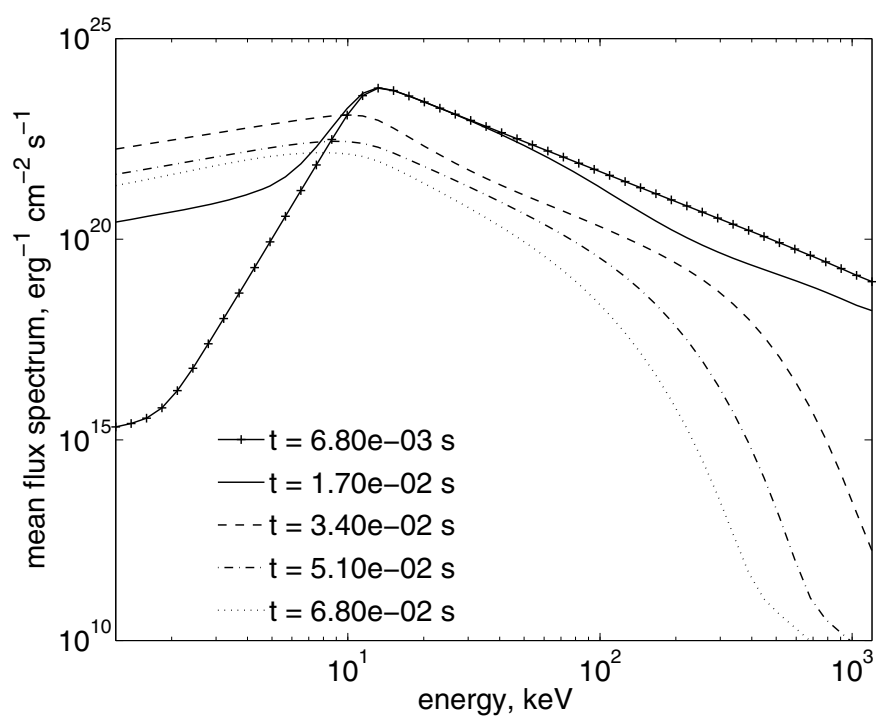

(d) collisions and convergence according to Eq. (39)

Fig. 11. Mean flux spectra of the electrons injected as a short impulse. The beam parameters are the same as in Fig. 10.

too low to be noticeable. The heating profile is also narrower but higher and its maximum located higher in the atmosphere compared to the $\gamma=3$ case. When the electric field is taken into account (Fig. 13b), the heating becomes stronger at lower depths in the corona, in comparison to the pure collisional case (Fig. 13a), where it reaches a maximum in the upper chromosphere.

A more powerful beam with energy flux $10^{12} \mathrm{erg} \mathrm{cm}^{-2} \mathrm{~s}^{-1}$, obviously deposits more of its energy in the ambient atmosphere (Fig. 14) than a beam with energy flux $10^{10} \mathrm{erg} \mathrm{cm}^{-2} \mathrm{~s}^{-1}$. Two maxima are clearly evident in the heating function profile - one in the chromosphere, another in the corona. If the magnetic convergence is absent, the chromospheric heating is much stronger (Fig. 14a). On the other hand, if the convergence is taken into account, then only about $10 \%$ of electrons can reach the chromosphere. Thus, the heating beneath the transition region is reduced by an order of magnitude, while the coronal heating remains nearly the same as in the case of the constant magnetic field (Fig. 14c). We note that in this case a different hydro-dynamic model is used to estimate the density and temperature of the ambient plasma. The discontinuity at the depth of $10^{20} \mathrm{~cm}^{-2}$ (Fig. 14) is caused by a sharp increase in the ambient plasma density (see Fig. 1), which apparently corresponds to the transition region.

It can be noted that the evolution time of an electron impulse is longer for a stronger beam. This is because of the lower density of the ambient plasma, which leads to a longer relaxation time as shown in Sect. 4.1.

\subsection{Bursts of hard X-ray emission}

To be able to compare with observations, we calculate the intensity of hard X-ray emission produced by the injection of a short electron beam. The bremsstrahlung cross-sections are taken in the relativistic form (see Gluckstern \& Hull 1953). Figure 15a shows the time profile of hard X-ray intensity produced by an impulse of length $\delta t_{\mathrm{e}}=1.7 \mathrm{~ms}$. The timescale of the hard $\mathrm{X}$-ray impulse, $\delta t_{\mathrm{hxr}}$, is about $20 \mathrm{~ms}$, which is determined by the relaxation time of the atmosphere $t_{\mathrm{r}} \approx 70 \mathrm{~ms}$ established 


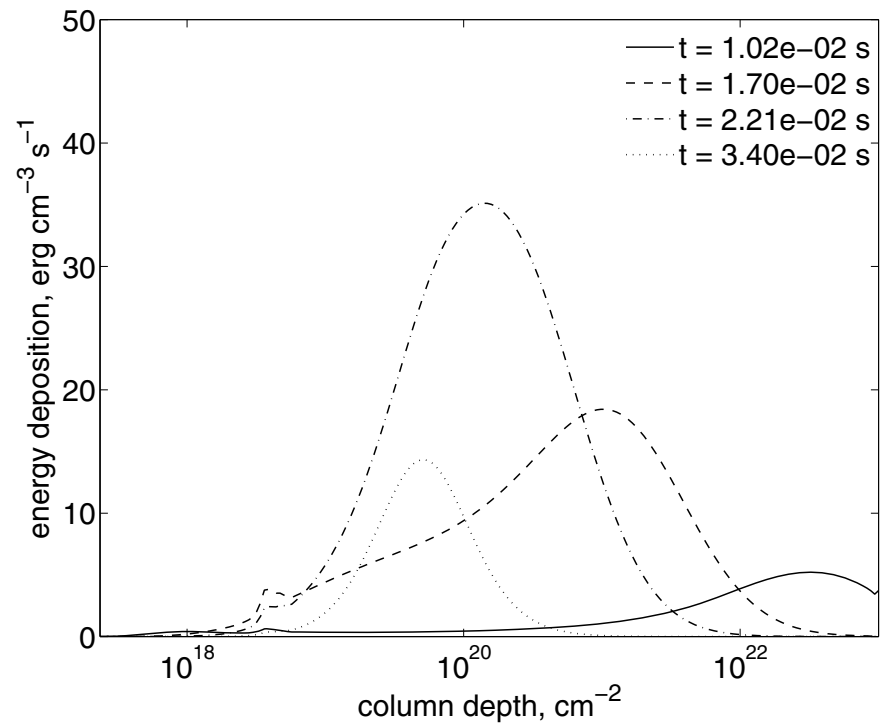

(a) collisions

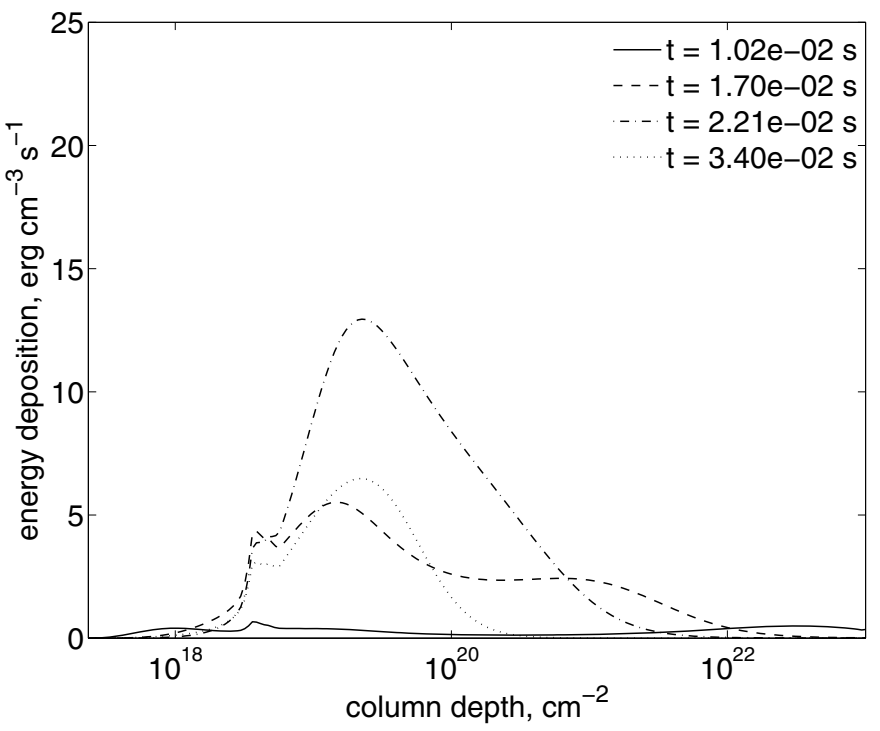

(c) collisions and convergence

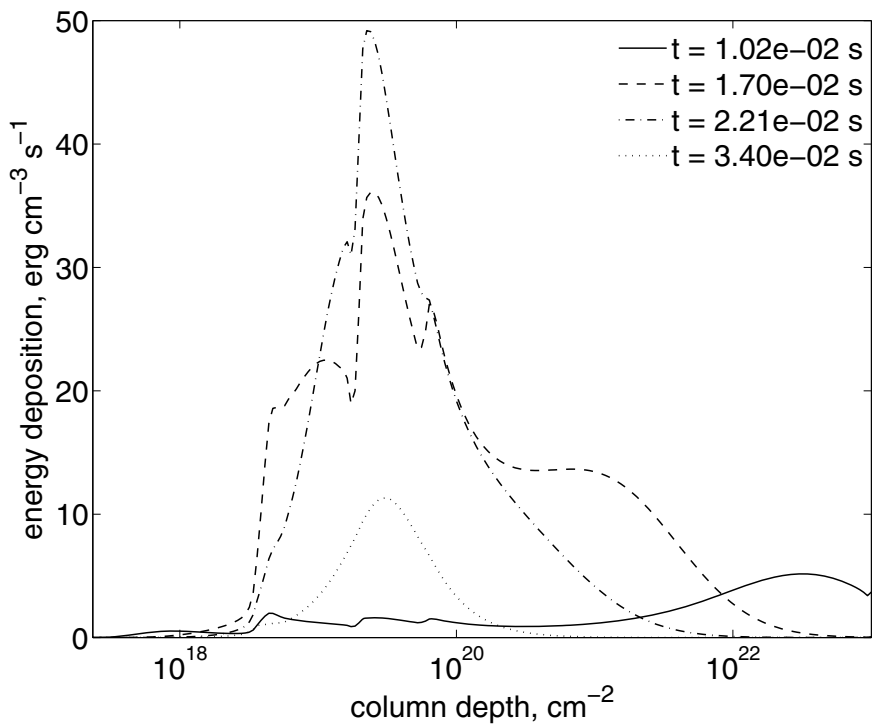

(b) collisions and electric field

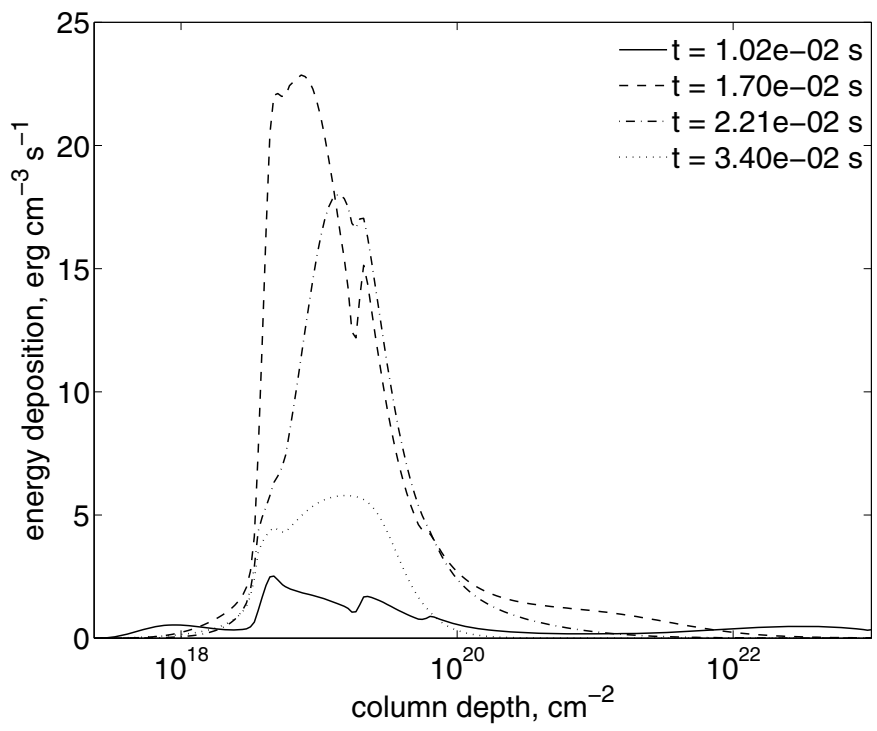

(d) collisions, convergence and electric field

Fig. 12. Energy deposition by a beam impulse. The beam parameters are the same as in Fig. 10 and the magnetic convergence is given by Eq. (36).

earlier in Sect. 4.1. This timescale agrees closely with observations (Charikov et al. 2004). Further simulations show that as long as $\delta t_{\mathrm{e}} \ll \delta t_{\mathrm{hxr}}$, the hard X-ray timescale depends only on the atmosphere parameters and does not depend on the length of the initial electron impulse.

The evolution in the spatial profile of the hard X-ray intensity (Fig. 15b) resembles that of the energy deposition (see Fig. 12b). The emission starts at the bottom when high energy electrons reach this depth and gradually moves upwards. After reaching the depth $\sim 2 \times 10^{19} \mathrm{~cm}^{-2}$, the intensity of the emission decreases and the emission finally vanishes.

\section{Conclusions}

By solving the time-dependent Fokker-Planck equation numerically, one is able to study the temporal evolution of the electron beam precipitation in the solar atmosphere and evaluate the relaxation time required for the beam to reach the stationary regime. For the beam with energy flux $10^{10} \mathrm{erg} \mathrm{cm}^{-2} \mathrm{~s}^{-1}$, this relaxation time is $\sim 0.07 \mathrm{~s}$ and becomes longer by a factor of about 3 (or $\sim 0.2 \mathrm{~s}$ ) for a beam with energy flux $10^{12} \mathrm{erg} \mathrm{cm}^{-2} \mathrm{~s}^{-1}$.

The effect of the self-induced electric field during the stationary beam injection is similar to that found in previous studies by Emslie (1980) and Zharkova \& Gordovskyy (2006). In particular, if the electric field is taken into account, then the maximum of the energy deposition profile is shifted upwards making the coronal heating stronger and the chromospheric heating weaker than in the case of pure collisional precipitation.

We considered different models of a converging magnetic field to study the effectiveness of the beam's electron refraction by a magnetic mirror. Magnetic field approximations used earlier by Leach \& Petrosian (1981) and McClements (1992) have the same spatial dependence in the corona and chromosphere. Even if the magnetic field in the photosphere is accepted to be 3 orders of magnitude higher than in the corona, these magnetic profiles are shown to affect only the high energy electrons of the beam. We propose a model where the magnetic field increases 


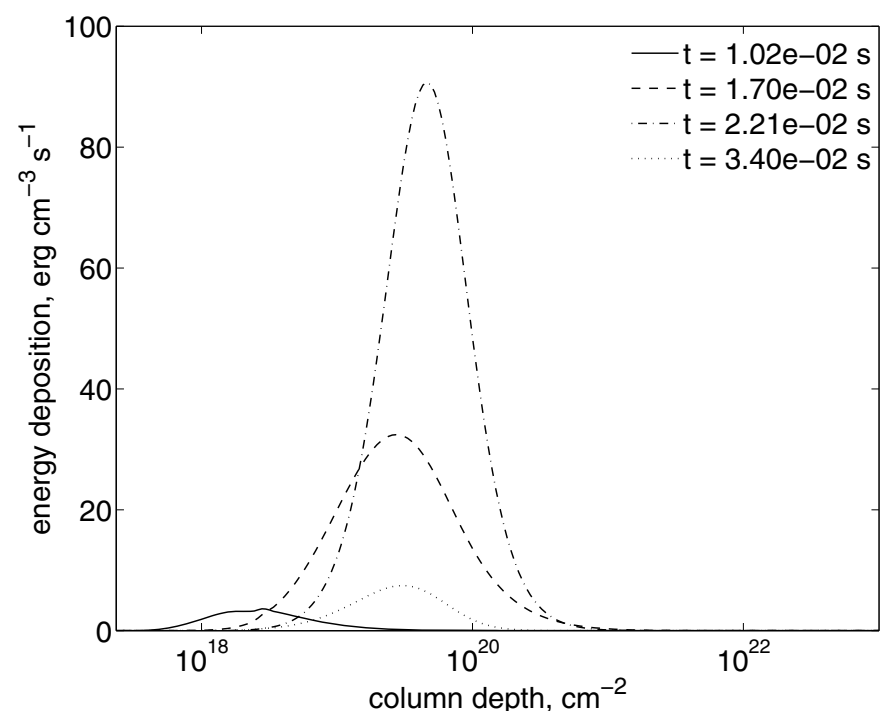

(a) collisions

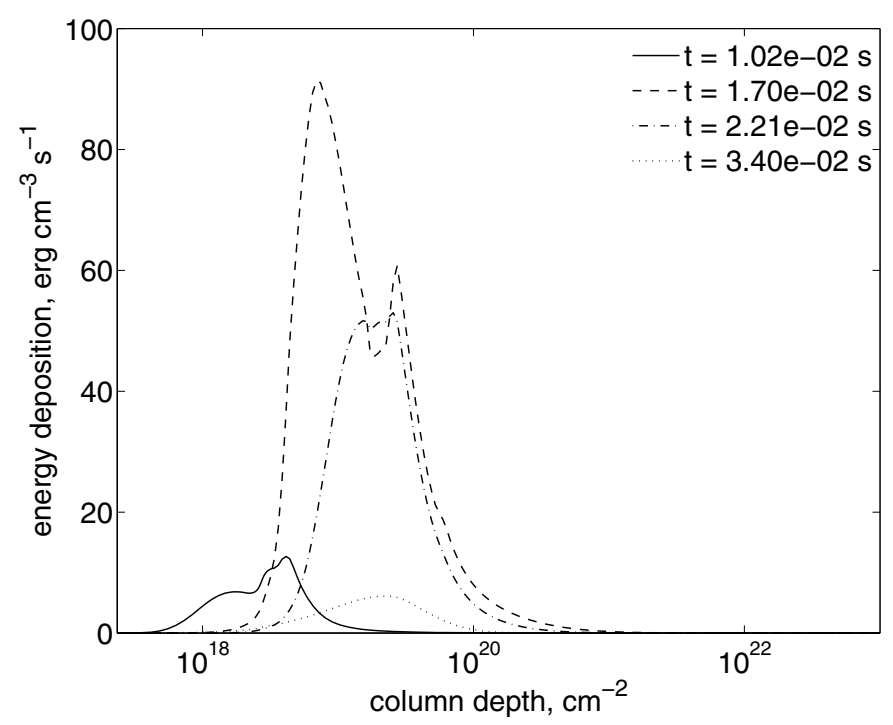

(b) collisions and electric field

Fig. 13. Energy deposition by the impulse of a beam with $\gamma=7$. The other beam parameters are the same as in Fig. 10.

exponentially with depth in the corona and becomes constant in the lower chromosphere. These magnetic field variations can affect the entire energy spectrum of electrons, while the ratio of photospheric to coronal magnetic fields can be as low as 23 . Since the converging magnetic field returns many electrons back to the source, the heating due to collisions and electric field is reduced by $70 \%$ in comparison with the constant magnetic field. We also considered the model based on indirect measurements of the magnetic field in the solar atmosphere. This magnetic field variation can also affect electrons of all energies and reduce the collisional heating by $20 \%$ compared to the case of a constant magnetic field profile.

The further study is dedicated to the impulsive injections of electrons. In the simulation of impulsive injection, the length of the impulse is chosen to be $1.7 \times 10^{-3} \mathrm{~s}$, which is much shorter than the relaxation time. It was found that the effect of the electric field is considerably smaller for the short impulse than for the steady injection.

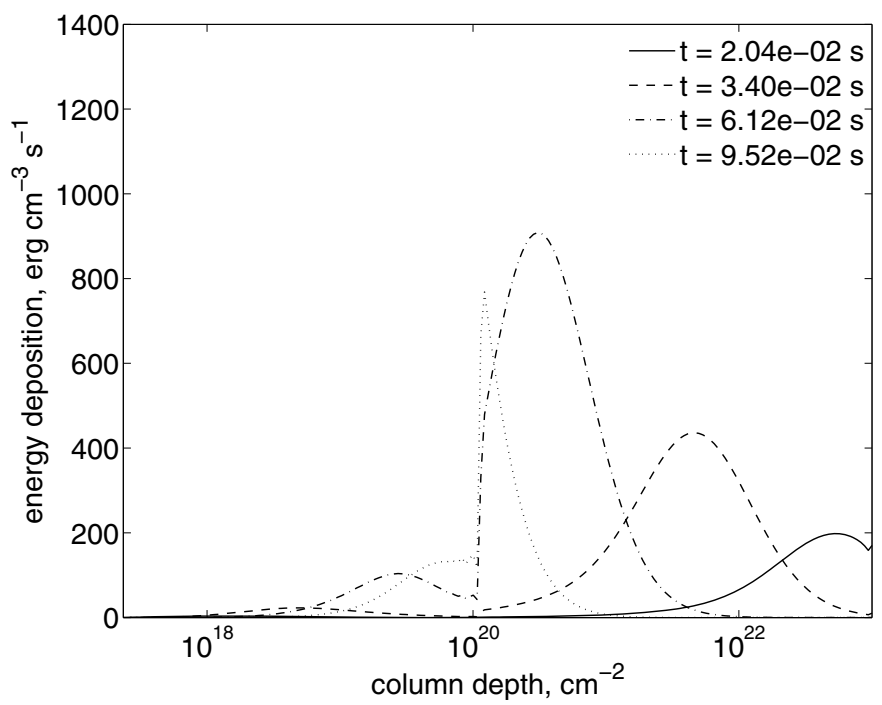

(a) collisions

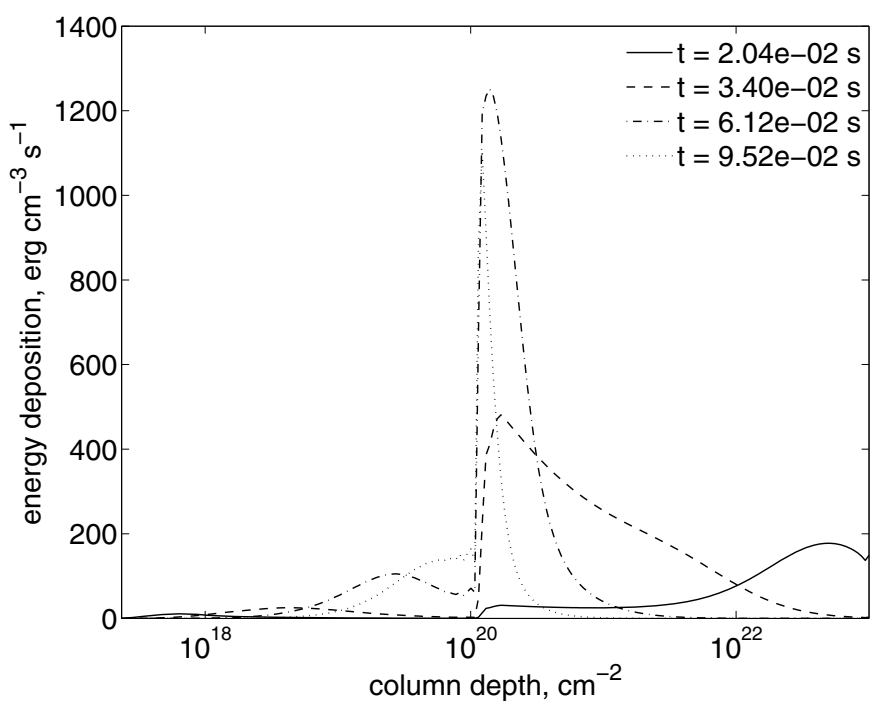

(b) collisions and electric field

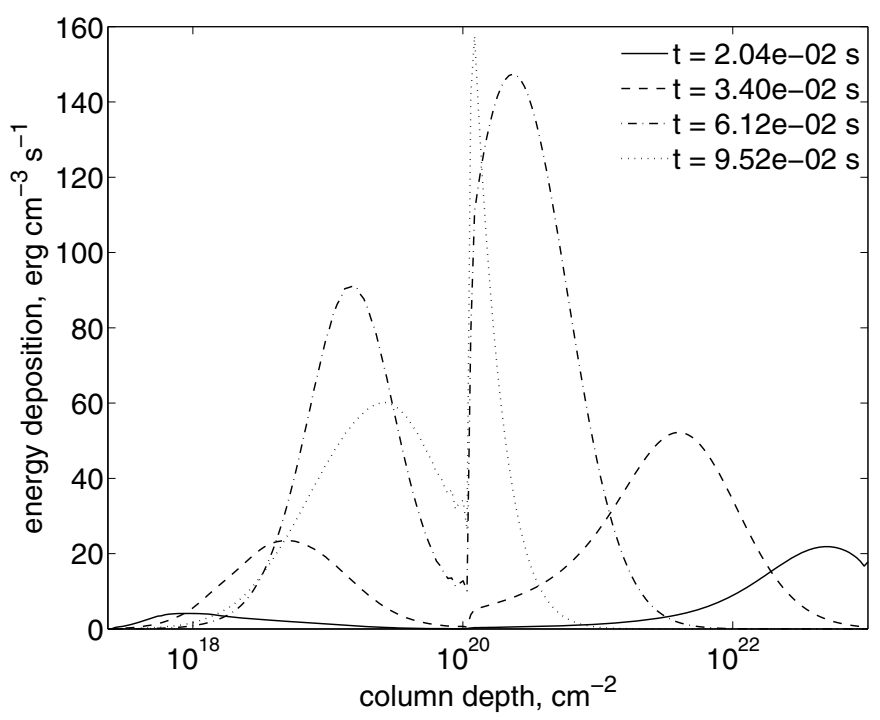

(c) collisions and convergence

Fig. 14. Energy deposition of the beam impulse with the energy flux $10^{12} \mathrm{erg} \mathrm{cm}^{-2} \mathrm{~s}^{-1}$. The other beam parameters are the same as in Fig. 10 and the magnetic convergence is given by Eq. (36). 


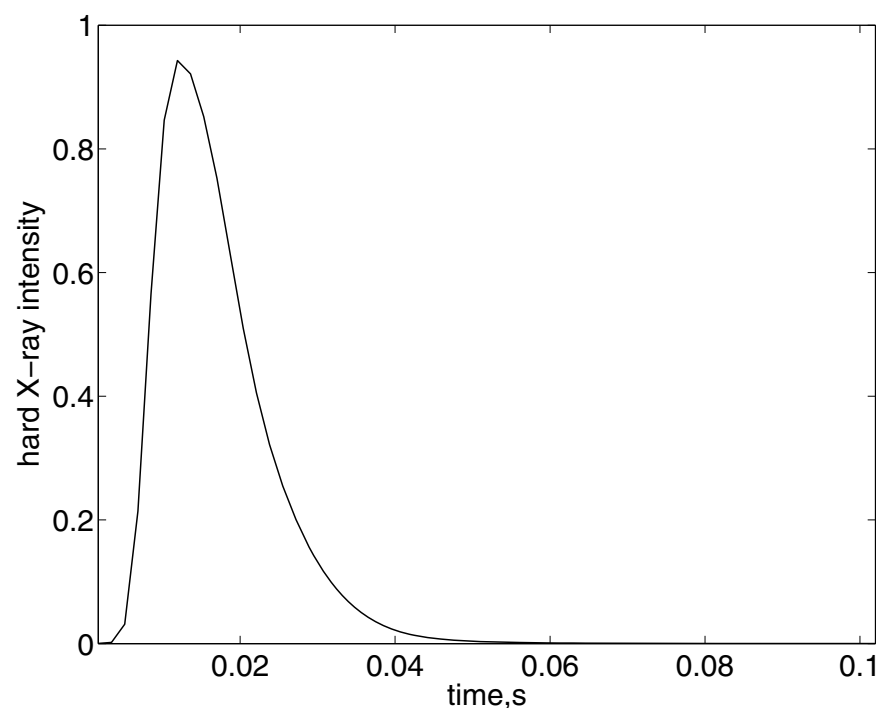

(a)

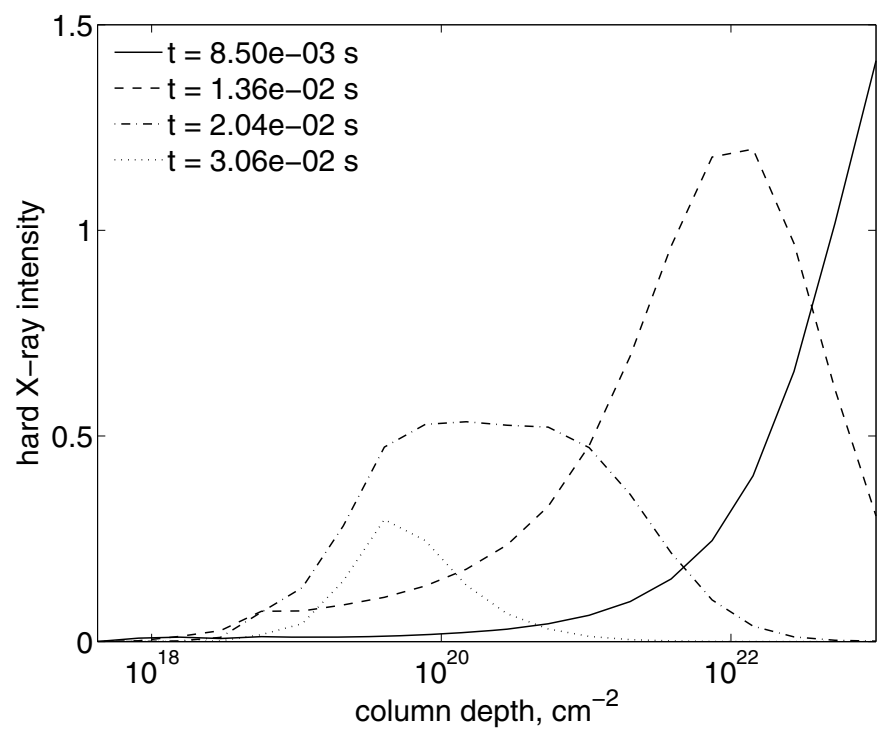

(b)

Fig. 15. Intensity of the hard X-ray emission burst (in arbitrary units): integrated over depth as a function of time a) and temporal evolution of the spatial profile b). Beam parameters are the same as in Fig. 10.

The initial energy spectrum of the injected impulse was assumed to be a power law. It was shown that during the evolution of the impulse the power law index increases in time. For example, if the joint effects of the collisions and magnetic convergence are taken into account, the initial power law index of 3 can increase to 8 resembling some quasi-thermal distributions.

The energy deposition profile is shown to depend on the initial power law index. If the energy spectrum is hard $(\gamma=3)$, the heating starts at the bottom end of the system due to the high energy electrons. In the case of a soft $(\gamma=7)$ impulse, the number of high energy particles is too low to produce any noticeable heating of the deep layers. On the other hand, the higher layers are heated more effectively because of the higher number of low energy electrons in the softer beam.

We also compared the evolution of beams with different intensities. It was found that the difference in this case is mostly caused by the different density and temperature profiles (see Fig. 1) taken from the hydrodynamic model (Zharkova \& Zharkov 2007). For example, the timescale of the impulse evolution is longer the more intense the beam.

If the timescale of the electron impulse is short enough, then the timescale of the hard X-ray emission is determined by the reaction of the atmosphere. Thus, it is of the same order of magnitude as the relaxation time, which, in turn, is of the order of $10 \mathrm{~ms}$ and longer. This means that shorter electron impulses cannot be detected by hard X-ray observations.

Acknowledgements. This research is funded by the Science Technology and Facility Council (STFC) project PP/E001246/1.

\section{References}

Brosius, J. W., \& White, S. M. 2006, ApJ, 641, L69

Brown, J. C. 1971, Sol. Phys., 18, 489

Brown, J. C., Karlický, M., Mandzhavidze, N., \& Ramaty, R. 2000, ApJ, 541, 1104

Brown, J. C., Emslie, A. G., \& Kontar, E. P. 2003, ApJ, 595, L115

Charikov, Y. E., Dmitriyev, P. B., Koudriavtsev, I. V., et al. 2004, in Multi-

Wavelength Investigations of Solar Activity, ed. A. V. Stepanov, E. E. Benevolenskaya, \& A. G. Kosovichev, IAU Symp., 223, 429

Diakonov, S. V., \& Somov, B. V. 1988, Sol. Phys., 116, 119

Emslie, A. G. 1980, ApJ, 235, 1055

Fisher, G. H., Canfield, R. C., \& McClymont, A. N. 1985, ApJ, 289, 414

Fletcher, L., Hannah, I. G., Hudson, H. S., \& Metcalf, T. R. 2007, ApJ, 656, 1187

Gluckstern, R. L., \& Hull, M. H. 1953, Phys. Rev., 90, 1030

Grechnev, V. V., Kurt, V. G., Chertok, I. M., et al. 2008, Sol. Phys., 252, 149

Grigis, P. C., \& Benz, A. O. 2006, A\&A, 458, 641

Holman, G. D., Sui, L., Schwartz, R. A., \& Emslie, A. G. 2003, ApJ, 595, L97

Kane, S. R., Anderson, K. A., Evans, W. D., Klebesadel, R. W., \& Laros, J. G. 1980, ApJ, 239, L85

Kiplinger, A. L., Dennis, B. R., Frost, K. J., Orwig, L. E., \& Emslie, A. G. 1983, ApJ, 265, L99

Kontar, E. P., MacKinnon, A. L., Schwartz, R. A., \& Brown, J. C. 2006, A\&A, 446, 1157

Kontar, E. P., Hannah, I. G., \& MacKinnon, A. L. 2008, A\&A, 489, L57

Krucker, S., Saint-Hilaire, P., Christe, S., et al. 2008, ApJ, 681, 644

Kundu, M. R., Nindos, A., \& Grechnev, V. V. 2004, A\&A, 420, 351

Lang, K. R., Willson, R. F., Kile, J. N., et al. 1993, ApJ, 419, 398

Leach, J., \& Petrosian, V. 1981, ApJ, 251, 781

Lin, R. P., Krucker, S., Hurford, G. J., et al. 2003, ApJ, 595, L69

McClements, K. G. 1990, A\&A, 234, 487

McClements, K. G. 1992, A\&A, 258, 542

Nagai, F., \& Emslie, A. G. 1984, ApJ, 279, 896

Samarskii, A. A. 2001, Monographs and Textbooks in Pure and Applied Mathematics, The theory of difference schemes (New York: Marcel Dekker Inc.), 240, translated from the Russian

Siversky, T. V., \& Zharkova, V. V. 2009, J. Plasma Phys., in press

Somov, B. V., Spektor, A. R., \& Syrovatskii, S. I. 1981, Sol. Phys., 73, 145

Somov, B. V., Sermulina, B. J., \& Spektor, A. R. 1982, Sol. Phys., 81, 281

Syrovatskii, S. I., \& Shmeleva, O. P. 1972, SvA, 16, 273

van den Oord, G. H. J. 1990, A\&A, 234, 496

Zharkova, V. V., \& Kobylinskij, V. A. 1992, Kinematics and Physics of Celestial Bodies, 8, 34

Zharkova, V. V., \& Gordovskyy, M. 2005a, Space Sci. Rev., 121, 165

Zharkova, V. V., \& Gordovskyy, M. 2005b, A\&A, 432, 1033

Zharkova, V. V., \& Gordovskyy, M. 2006, ApJ, 651, 553

Zharkova, V. V., \& Zharkov, S. I. 2007, ApJ, 664, 573

Zharkova, V. V., Brown, J. C., \& Syniavskii, D. V. 1995, A\&A, 304, 284

Zharkova, V. V., Kuznetsov, A. A., \& Siversky, T. V. 2009, A\&A, in press 